\title{
The role of climatic forcings in variations of Portuguese temperature: A comparison of spectral and statistical methods
}

\author{
Anna L. Morozova ${ }^{a, *}$, Tatiana V. Barlyaeva ${ }^{\mathrm{b}}$ \\ ${ }^{a}$ CITEUC-Centre for Earth and Space Research of the University of Coimbra, University of Coimbra, Almas de Freire, Sta. Clara, Coimbra 3040 - 004, Portugal \\ ${ }^{\mathrm{b}}$ Laboratoire d'Astrophysique de Marseille, UMR 7326, CNRS and Aix-Marseille Université, 13388, Marseille, France
}

\section{A R T I C L E I N F O}

\section{Article history:}

Received 5 October 2015

Received in revised form

21 January 2016

Accepted 4 February 2016

Available online 6 February 2016

Keywords:

Regional temperature variations

Solar activity and climate

Wavelet analysis

Multiple regression models

\begin{abstract}
A B S T R A C T
Monthly series of temperature parameters measured by three Portuguese meteorological stations from 1888 to 2001 were used to study the effect of different climatic forcings. Three types of external forcings were considered: anthropogenic greenhouse gases and aerosols, volcanic aerosols, and solar and geomagnetic activity variations. Long-term variations of the temperature and other parameters with characteristic periods of decades were studied by various methods including the seasonal-trend decomposition based on LOESS (LOcally wEighted regreSSion), correlation and multiple regression analyses, and wavelet/wavelet coherence analyses. Obtained results confirm the statistical dependence of the temperature variations on the volcanic and the anthropogenic influence as well as variability that can be associated with the solar activity impact. In particular, surprisingly strong bi-decadal cycles were observed in temperature series whereas the observed decadal cycles are weaker and transient. Another interesting finding is the apparent non-stationarity of the relations between the solar and atmospheric parameters probably related to periods of strong/weak global circulation or frequent/occasional volcanic eruptions or interaction between the external forcing and internal atmospheric variability.
\end{abstract}

(c) 2016 Elsevier Ltd. All rights reserved.

\section{Introduction}

Investigations of the external forcings effect on climate variability starts long ago. Various types of mathematical methods (statistical analyses, numerical simulations, time-frequency analyses etc.) were applied to a wide number of atmospheric parameters like temperature, pressure, precipitation, wind speed and wind direction etc. to understand the roles played in their variations by different external climatic forcings: anthropogenic gases and aerosols, volcanic aerosols, solar irradiance, and solar and geomagnetic activity as well as some internal variabilities like atmospheric circulation and oceanic impact. Some studies show non-stationarity of forcings control. For example, (Meehl et al., 2003, Hegerl et al., 2007, 2011, Lockwood and Fröhlich, 2007; Lean and Rind, 2008) showed that during the last centuries temperature variations until the beginning of the 20th c. can be explained using mostly volcanic and solar forcings, but the rapid increase of the temperature at the end of this century needs other source anthropogenic greenhouse gases. Lockwood (2012) emphasizes the differences between the global and the regional climate

\footnotetext{
* Corresponding author.

E-mail addresses: anna_m@teor.fis.uc.pt,

annamorozovauc@gmail.com (A.L. Morozova),

tatiana.barlyaeva@lam.fr (T.V. Barlyaeva).
}

sensitivity to forcings. The regional climate has stronger response and larger spatial variability in the sign and amplitude of such response. Differences in the reaction of the regional climatic series to the external forcings (in particular, solar activity and geomagnetic field variations) were found by (Usoskin et al., 2010). Their study highlights the difference (and its sources) in the response of climatic parameters form different latitudinal zones (polar, middle latitudinal and equatorial) to the solar forcing.

There is also a possibility that external factors can affect some atmospheric parameters twice: directly and indirectly. For example, the total solar irradiance variations affects lower atmosphere temperature both directly (changes of the radiation balance) and indirectly through (e.g.) changes of the global circulation patterns resulting in warm/cold air advection, changes of cloudiness and precipitation regimes. Same can be said for the volcanic and anthropogenic influences. The presence of a combined effect of the solar and volcanic activities on the 11-yr periodicity in the global Earth temperature was detected by (Barlyaeva et al., 2009). Further, the difference in a response of the regional air-surface temperatures to the solar, volcanic and geomagnetic forcings at the decadal (11-yr and 22-yr) timescale was shown in (Barlyaeva, 2013). In particular, it was found that the response of temperature to the solar forcing for distant climate zones differs from each other, changes with time and depends on the presence of other influencing factors. The widespread response was also observed to 
the 22-yr oscillations of the geomagnetic activity. The change of the phase in correlation between solar and atmospheric parameters is probably related to the interaction between the periodic external forcing and internal oscillations of the atmosphere or the atmosphere-ocean system was detected by, e.g., (Raspopov et al., 2004; Gusev and Martin, 2012) (see also references therein). Such phase changes can be generated in a non-linear climate system which has two quasi-stable states under the influence of a weak external signal (Tobias and Weiss, 2000 and references therein).

The climatic variations of the south-western Europe and Iberian Peninsula were studied previously using both observed or reconstructed data and simulations by the state-of-art atmospheric models (e.g., del Río et al., 2012; Gámiz-Fortis et al., 2011; GómezNavarro et al., 2010, 2012, 2013; Hegerl et al., 2011). In particular, Gómez-Navarro et al. (2012) modeled climate variability of this region over the last millennium in response to the externals forcings of anthropogenic gases $\left(\mathrm{CO}_{2}\right.$, methane, $\left.\mathrm{NO}\right)$, volcanoes and total solar irradiance (TSI) variations due to solar activity cycles. del Río et al. (2012) analyzed measured temperature parameters over Spain in the second half of the 20th c. They found significant differences in the time trends of the daily minimum (Tmin) and maximum (Tmax) temperatures leading to specific trends in the daily temperature range (DTR). They indentified the main external forcings responsible for the observed trends such as greenhouse gases, solar radiation, and anthropogenic aerosols and paid attention to the internal atmospheric variability due to the changes in circulation patterns (mostly the North-Atlantic Oscillation, NAO).

In this study we used regional monthly temperature data measured by three meteorological stations located in a relatively small area of the Portuguese west region. The original temperature series are of a monthly resolution allowing us to study not only the mean annual climate response to the forcings but also its seasonal variations. These historical series (described in Section 2) were recently presented to the scientific community after the homogenization procedure done in the frame of the of the FP7 project ERA-CLIM. They cover a period from middle/end of the $19^{\text {th }} \mathrm{c}$. to the beginning of the 21 th $\mathrm{c}$. As to the parameters used to characterize the external atmospheric forcings, we tried to use as much of direct measurements or reconstructions based on direct measurements as possible. Another advantage of our study is that the data were analyzed using two different mathematical approaches: statistical (based on the correlation and regression analysis) and spectral (using wavelet and wavelet phase/coherence analysis). The results obtained by one method (e.g., wavelet coherence/ phase analysis) can be cross-checked by others (e.g., running correlation with different time lags) to test or enhance the statistical significance of obtained results. Different mathematical methods applied to the data help to look at the problem from various points of view, to see different properties and different connections. The similarity of the results of a number of various mathematical approaches could be used as an additional way to ensure the reliability of the obtained results, since different methods can have different sensitivities to specific properties of the data. Certainly, any statistical relations do not guarantee real physical connections, even if we know that some of forcings taken into account do have physical ways to drive climatic variations, and others can be involved in mechanisms which are currently discussed.

The paper is organized as follows: Section 1 presents modern state of the art and briefly gives overview of the paper. Sections 2 and 3 contain the descriptions of the data sets in use and the applied mathematical methods, respectively. In Section 4 we analyze footprints of different climatic forcings in the regional temperature variations. We also compare results produced by the different mathematical methods and discuss sources of the multi- decadal variability of the temperature series. Section 5 gives summary on the obtained results. Appendices A and B present the detailed description of one of the mathematical methods we use in this study and which is not yet widely used: the seasonal-trend decomposition based on LOESS.

\section{Data}

A number of monthly and annually averaged data were used in this study to analyze variations of the climate in the studied region and their possible forcings. Brief descriptions and pre-processing procedures of these datasets are presented below. Please note, that we use here the term "normalization" for a linear transform of a series to have zero mean and unit variance (standard deviation).

\subsection{Temperature}

In this study we took advantage of a newly available homogenized data set which consist of sets of 12 monthly series for each of the following parameters: monthly means of the daily minimum (Tmin) and daily maximum (Tmax) temperatures measured by three Portuguese stations, and calculated from them monthly means of the mean daily temperature (averT) and daily temperature range (DTR).The stations are

1. Porto $\left(\varphi=41^{\circ} 08^{\prime} \mathrm{N}, \lambda=8^{\circ} 36^{\prime} \mathrm{W}\right.$, from 1888 to 2001$)$;

2. Coimbra ( $\varphi=40^{\circ} 12^{\prime} \mathrm{N}, \lambda=8^{\circ} 25^{\prime} \mathrm{W}$, from 1865 to 2005$)$;

3. Lisbon ( $\varphi=38^{\circ} 43^{\prime} \mathrm{N}, \lambda=9^{\circ} 09^{\prime} \mathrm{W}$, from 1856 to 2008$)$.

Non-climatic breaks that existed in the raw series were identified during the homogenization procedure and the series were corrected using available metadata and statistical homogeneity tests. For the full description of homogenization procedure see (Morozova and Valente, 2012). The data are available at http://doi. pangaea.de/10.1594/PANGAEA.785377. Recently, this data set was used in a study of the effect of the atmospheric aerosol variations on regional climate in the last quarter of the 20th c. (Morozova and Mironova, 2015). Here we use this data set in its full length (more than 100 years) to find the effect of different external forcings on the Portuguese climate. The monthly series were used to calculate the seasonally and annually averaged series (hereafter, "seasonal series" and "annual series" (An), respectively): December, January and February for winter (Wi), March, April and May for spring (Sp), June, July and August for summer (Su), and September, October and November for autumn (Au) series.

To remove individual features of climatic variations from the stations' data series (two coastal stations - Porto and Lisbon, and a station located in an elevated region at $\sim 40 \mathrm{~km}$ from the coast Coimbra) and obtain a series suitable for the analysis of the regional climate we averaged these three series using the Principal Component Analysis (PCA). PCA is a well known method to extract independent spatial-temporal modes of variability (principal components, PCs) when a number of series of the same parameter from different stations (grid points) are used. This method also estimates the explained variances for each of extracted modes. The first principal component (PC1) accounts for as much of the common variability in the original data as possible, the second $\mathrm{PC}$ accounts for as much of the remaining common variability and so forth. The PCs are orthogonal and conventionally non-dimensional, and all series are subjected to the PCA in the form of deviations from the each series time mean. The full descriptions of the method and its application to the analysis of different meteorological series can be found in (e.g.) (Bjornsson and Venegas, 1997, Hannachi et al., 2007; Shlens, 2009). In our study this technique was used as an averaging procedure extracting the 
a)
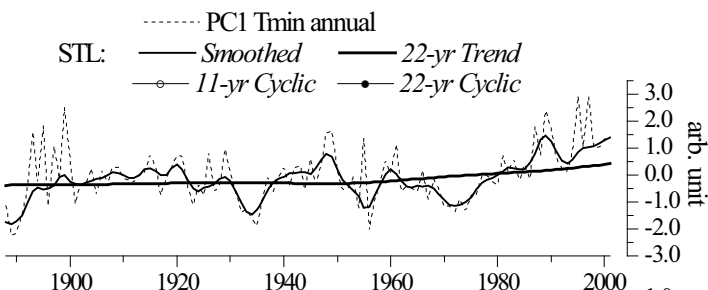

b)
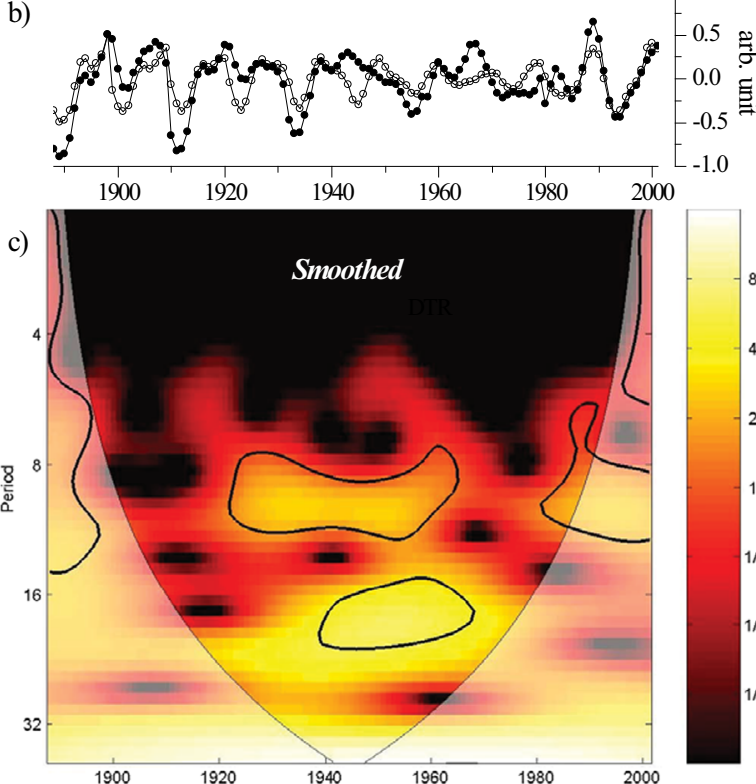

g)

h)

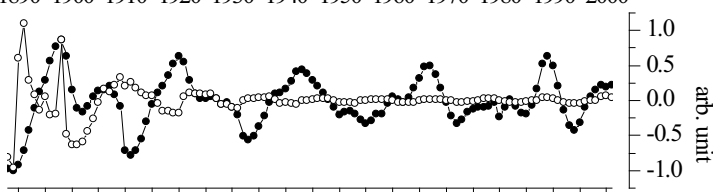

189019001910192019301940195019601970198019902000

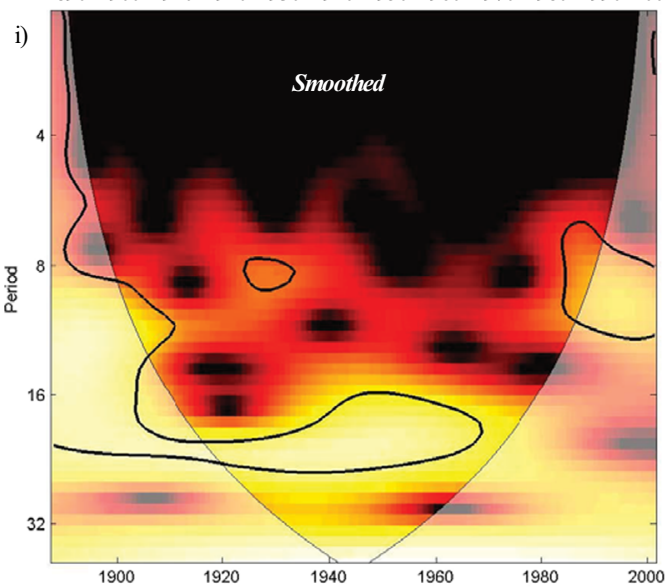

d)

e)
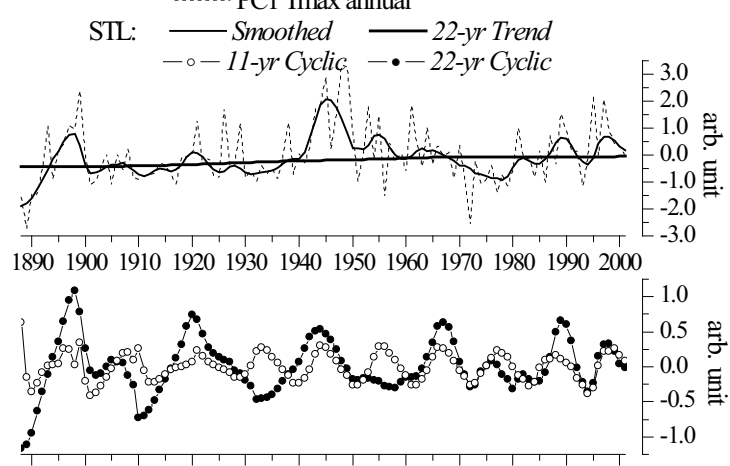

189019001910192019301940195019601970198019902000

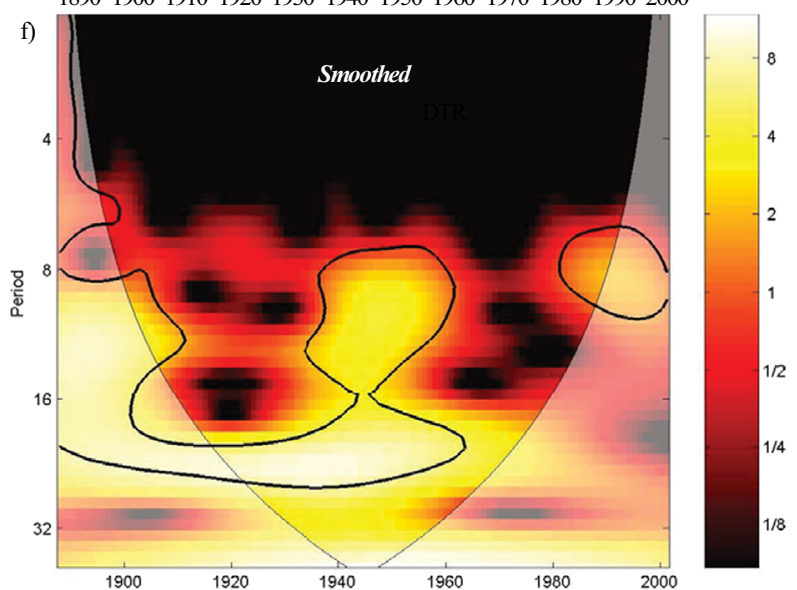

j)

STL: $\quad$ Smoothed $-22-y r$ Trend

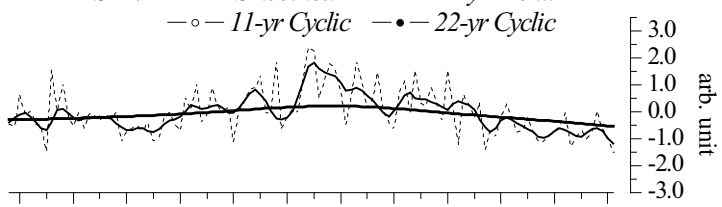

k)

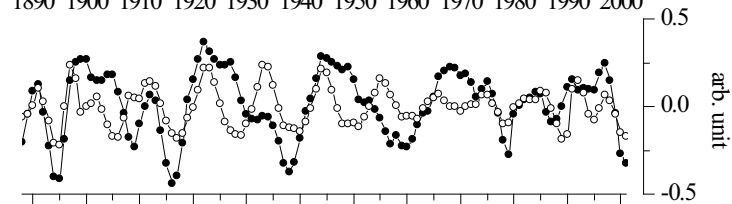

189019001910192019301940195019601970198019902000

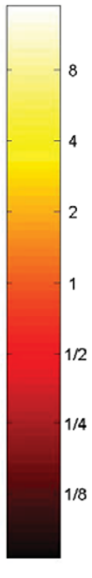

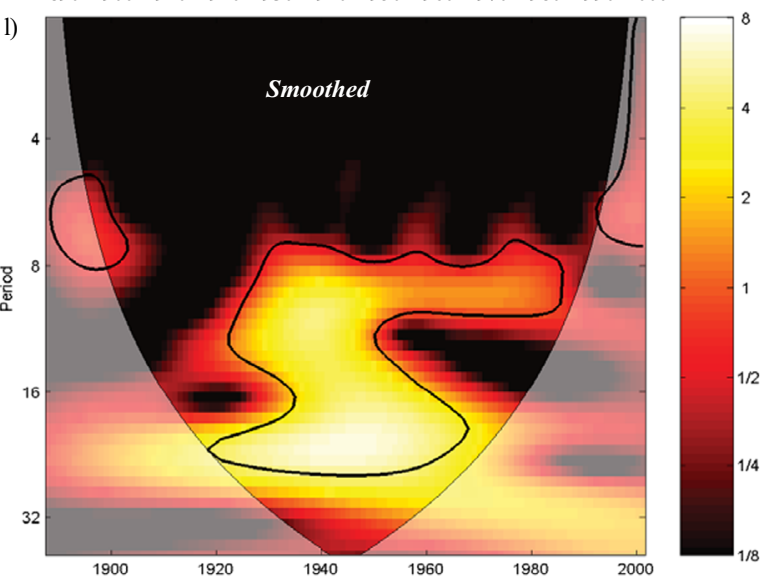

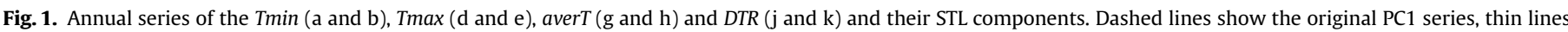

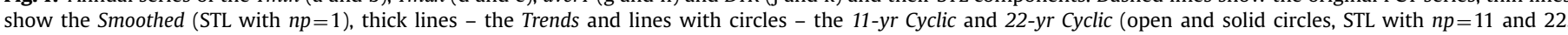

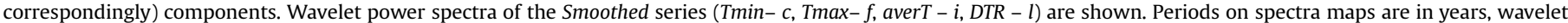
power amplitudes are non-dimensional. 
common variability from a number of similar data sets and smoothing original series (Hannachi et al., 2007). The obtained PC1s of the temperature series account for $88-95 \%$ of the variability of the original Tmin series, $85-97 \%$ of Tmax, 91-96\% of averT, and $80-94 \%$ of DTR series variability, depending on the month. The PC1 temperature series are available from 1888 to 2001.

To save the space we choose to show here only the results obtained for the annual series. In most cases corresponding figures for the seasonal series are provided in the Supplementary Material. The annual series of all four temperature parameters are shown in Fig. 1(a, d, g, j) as thin dashed lines and the corresponding seasonal series are shown in the Supp. Material, Figs. S2S3 (dashed lines).

\subsection{Forcings}

Portuguese temperatures variations, as well as in other regions, result from an interaction of the effect of global agents (like anthropogenic warming, volcanic eruptions or modes of global atmospheric circulation) and local features (landscape, prevailing winds, proximity to the ocean coast etc.). In this study we focus only to the global external forcings leaving the internal variability of the atmosphere-ocean system out of the frame of the study. The main goal is to estimate the part of the variability of the Portuguese temperature series that can be explained by the external influence alone and to define the input that can be assigned to each of the studied external forcings in particular.

Three types of external climatic forcings are considered:

1. Anthropogenic forcing described by the $\mathrm{CO}_{2}$ and the anthropogenic sulfate aerosols;

2. Volcanic forcing represented by the volcanic sulfate aerosols;

3. Solar forcing characterized by the sunspot numbers (SSN) and the geomagnetic indices (GAi).

To parameterize these forcings we used the following data sets available for the period from at least 1850 to about 2005. For some forcings the available data series have different lengths and cover different time intervals. However, to apply our methods (described in Section 3.2) we need for each of the forcing a single series covering a whole time interval from 1888 to 2001 - a period of temperature measurements - with at least annual time resolution. Consequently, for such forcings, composite series were created.

\subsection{1. $\mathrm{CO}_{2}$}

Three annual series of the measured and/or reconstructed annual level of $\mathrm{CO}_{2}$ atmospheric concentration obtained from different sources show strong coherence and were used to obtain a single composite series of sufficient length. First series is by (Robertson et al., 2001a, 2001b) and contains the atmospheric $\mathrm{CO}_{2}$ concentrations over period from 1500 to 1999 established on the basis of both the analysis of a fossil air trapped in ice cores and instrumental measurements. Second series is from (Mann et al., 2000a). It presents data reconstructed for the period 1610-1995 on the base of proxy and historical measurement series (Mann et al., 2000b). These two series are highly correlated but still have small differences, e.g., in the beginning of the 20th c. Both of them are calibrated with the Mauna Loa Observatory measurements. The third series is the measurements from Mauna-Loa Observatory available from 1959 to the present (http://www.esrl.noaa.gov/ gmd/ccgg/trends/mlo.html). The composite series was created because the original series are of different lengths and cover different time periods. The parent and the composite series are shown in the Supp. Material, Fig. S1a.

\subsubsection{Anthropogenic and volcanic sulfates}

Different data sets give us the information about the annual variability of total sulfate aerosol content. We used the following annual series:

1. The series of stratospheric aerosol optical thickness (AOD) calculated for the Northern Hemisphere to estimate the volcanic aerosol effect on climate for the Goddard Institute for Space Studies (GISS) climate simulations (http://data.giss.nasa.gov/ modelforce/strataer, see Sato et al., 1993 for the description). These data were used as a "volcanic sulfates" series.

2. Annual estimates of the anthropogenic global and regional sulfur dioxide emissions from the Historical Anthropogenic Sulfur Dioxide Emissions dataset by Socioeconomic Data and Applications Center (SEDAC) (Smith et al., 2011). This series was used as a control series for the anthropogenic sulfate aerosols content.

3. Measurements of the non-sea-salt sulfur (nssS) content in Greenland ice cores (McConnell et al., 2007a, 2007b).

All series are shown in the Supp. Material Fig. S1b. To obtain an "anthropogenic sulfates" series the nssS data were used. The lower envelope of this series strongly resembles the estimated anthropogenic sulfur dioxide emissions data (please see Supp. Material, Fig. S1b). The correlation coefficient between these series is 0.898 ( $p$ value $=0.005$, see Section 3.2 for the $p$ values definition) for the period 1888-1975 and 0.901 ( $p$ value $=0.003$ ) for the period 19752001. The effect of the strong volcanic eruptions is clearly seen in the series of nssS as sporadic peaks. Based on this analysis, we used the nssS content series as a proxy for the "anthropogenic sulfates" after removal of the scaled "volcanic sulfates". (see Fig. 2a and $\mathrm{d}$ ).

\subsubsection{Solar forcing}

Solar forcing is often attributed to the total solar irradiance (TSI) variations. TSI changes in the accordance with the solar activity cycles. These cycles are often parameterized by the sunspot numbers (SSN). In our study we did not used the TSI data directly on two reasons. First of all, there are a number of various TSI reconstructions which are based on different inputs and accepted physical mechanisms. All of them give plausible but slightly different TSI time variations. For the moment there is no a strong criterion to define which reconstruction is better. Secondly, we do not want to limit the solar activity effect only to the TSI variations. This is not only possible way for the Sun to affect the Earth's lower atmosphere. Here we just point out four other possible links between the solar activity variations and the Earth's climate: variations of the spectral solar irradiance (SSI), e.g., variations of the UV solar radiation; cosmic rays flux variations; geomagnetic field disturbances; atmospheric electric field variations. Last two have both 11-yr and 22-yr periodicities.

The ways for the solar and geomagnetic activity to affect climate are the subject of considerable debates among scientists. The least controversial mechanisms are those related to TSI and SSI variations already measured by a number of satellites (Fröhlich, 2012). There are both observations and models confirming SSI variations' effect on the stratosphere and its downward propagation (Palamara and Bryant, 2004; Lockwood, 2012). The other widely discussed range of mechanisms is linked to the charged cosmic rays particles and their probable connection to the atmospheric aerosol content and/or cloudiness (Voiculescu et al., 2006; Erlykin et al., 2010; Lockwood, 2012). In this connection we would like to mention that for the Iberian Peninsula the statistically significant correlation between the local low cloud cover and the solar UV flux was found by Voiculescu et al., 2006 and confirmed later by (Erlykin et al., 2010). The UV solar irradiance is highly 
a)

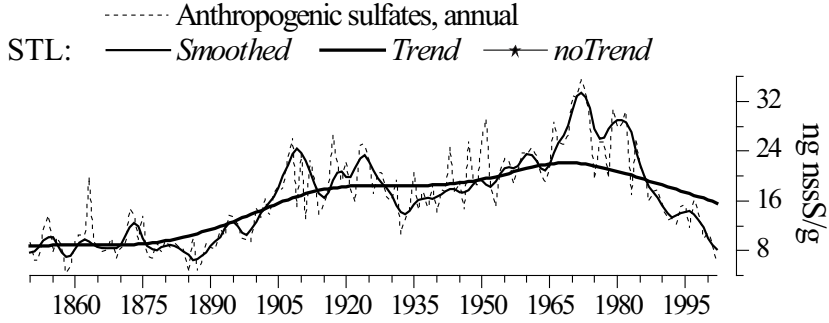

b)

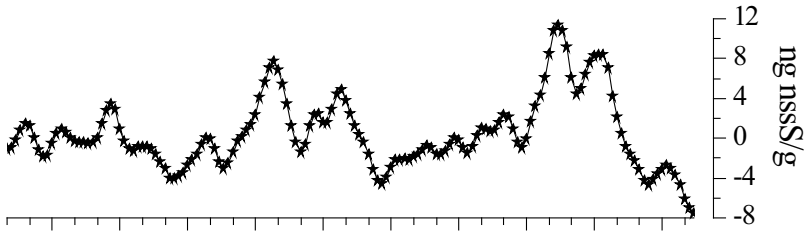

$\begin{array}{llllllllll}1860 & 1875 & 1890 & 1905 & 1920 & 1935 & 1950 & 1965 & 1980 & 1995\end{array}$

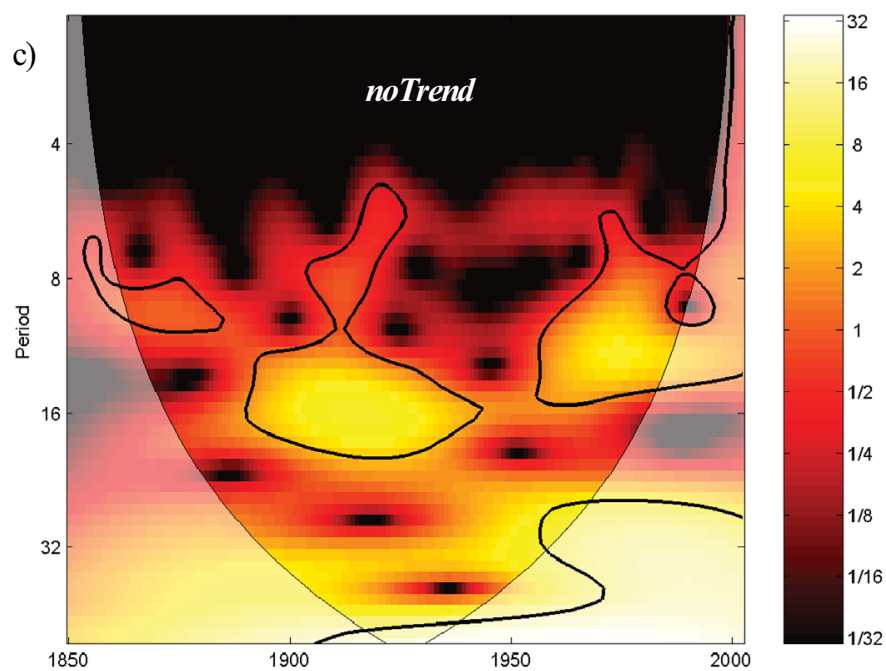

d)

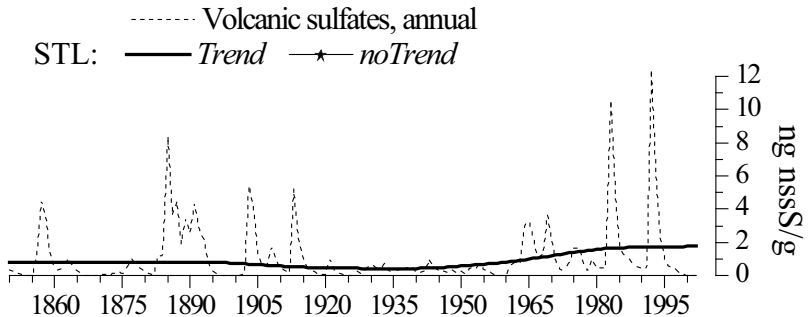

e)

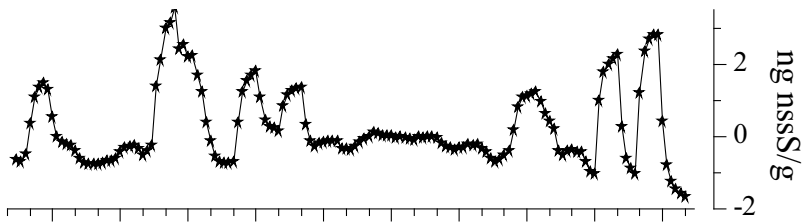

$\begin{array}{llllllllll}1860 & 1875 & 1890 & 1905 & 1920 & 1935 & 1950 & 1965 & 1980 & 1995\end{array}$

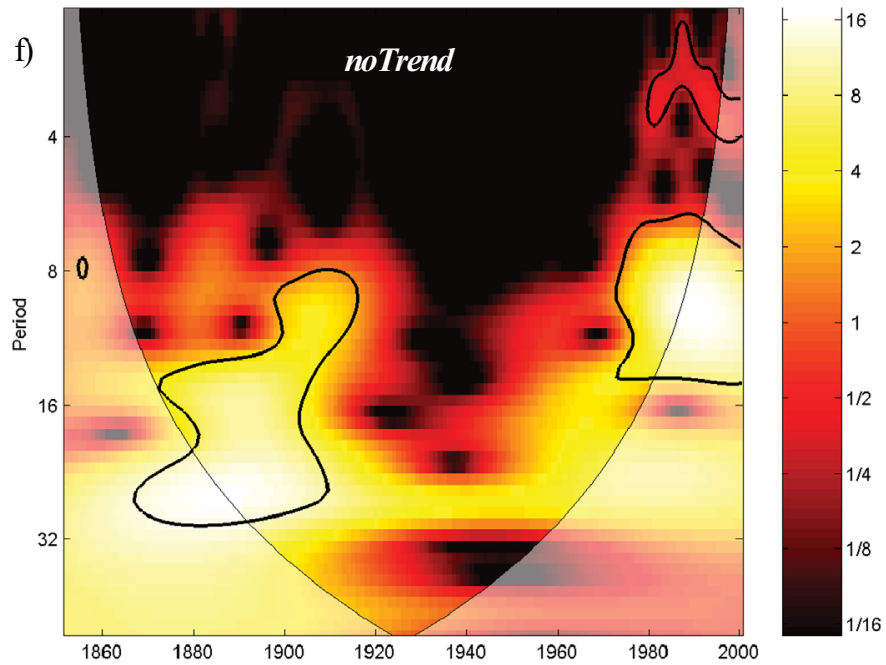

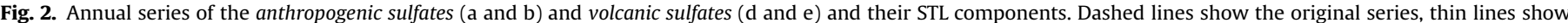

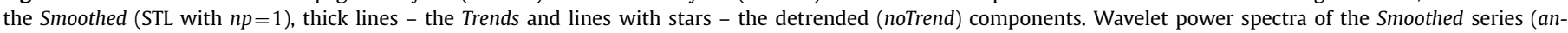
thropogenic sulfates $(c)$ and volcanic sulfates $(f)$ are shown. Periods on spectra maps are in years, wavelet power amplitudes are non-dimensional.

correlated with SSN. Besides, the relations between the atmospheric parameters and the geomagnetic indices suggest that the electric field variations (Lukianova and Alekseev, 2004; Tinsley, 2008; see also review (Lam and Tinsley, 2015) and references therein for the detailed discussion of the role that is played by the atmospheric electric field in cloud microphysics and other processes in the middle and low atmosphere) can be another possible agent connecting the solar and climatic variations through the aerosol and cloud formation processes.

Here we used the revised SSN series (Fig. 3a) for the time period from 1850 to 2005 that is currently available from the SILSO (Sunspot Index and Long-term Solar Observations) website (http:// www.sidc.be/silso/). SSN is not a direct measure of any physical agent that could influence the Earth's atmosphere but could be considered, in the frame of this study, as a proxy for the variations of other agents. The SSN series was recently revised to remove inhomogeneities accumulated through centuries of the sunspot observations by different observers and instruments and due to pervious corrections and re-scaling (Clette et al., 2014; 2015).

As to the geomagnetic field variations, we used a number of global geomagnetic indices. These indices are related to the geomagnetic variations caused by different sources in the magnetosphere and solar wind (Svalgaard et al., 2004). The aa index is the global index of geomagnetic activity which covers the interval from 1868 to the present time. It is available from the NGDC website (http://www.ngdc.noaa.gov/stp/spaceweather.html). The inter-hourly and inter-diurnal variations (IHV and IDV) annual indices are available from 1844 to 2010 and from 1872 to 2006, respectively, and describe the level of the geomagnetic activity in relation to the parameters of the solar wind. These indices are available (e.g.) from the Leif Svalgaard website (http://www.leif. org/research/) and are described in (Svalgaard and Cliver, 2005). These three indices show strong correlations $(r=0.71-0.995, p$ values $\leq 0.01$ ). The correlations are higher on the secular and slightly lower on the decadal time-scale (corresponding correlation coefficients can be found in the Supp. Material, Table 1).

Since one of the aims of this work is to statistically estimate on the whole the role of the solar forcing in the temperature variations of the studied region during the analyzed time period, we decided to use these geomagnetic indices to construct a single mean series (hereafter, GAi series) that preserves the main features of all the parent indices. To do this, each of the three series of the parent geomagnetic indices ( $a a, I H V, I D V)$ was normalized, then the mean series was calculated and standardized. The final standardized series is the GAi series used in the following analyses. The composite GAi series is highly correlated with the $a a, I H V$ and $I D V$ series. The series of all geomagnetic indices and $G A i$ as well as the corresponding correlation coefficients can be found in the Supp. Material, Fig. S1c-e (original, trends and detrended series, correspondingly). Only this single GAi series was used in the following 
a)

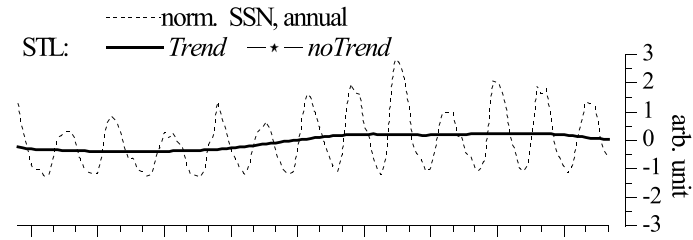

$\begin{array}{llllllllll}1875 & 1890 & 1905 & 1920 & 1935 & 1950 & 1965 & 1980 & 1995\end{array}$

b)

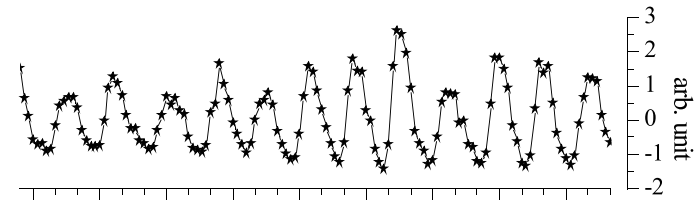

$\begin{array}{lllllllll}1875 & 1890 & 1905 & 1920 & 1935 & 1950 & 1965 & 1980 & 1995\end{array}$

c)

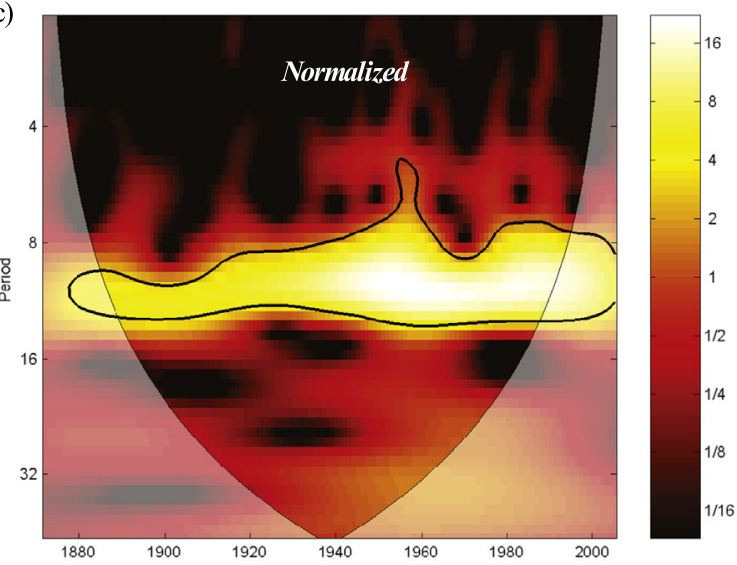

g)

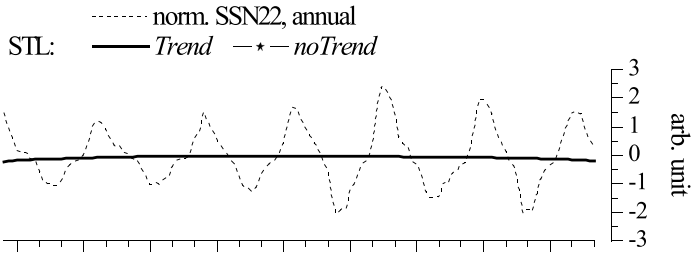

$\begin{array}{lllllllll}1875 & 1890 & 1905 & 1920 & 1935 & 1950 & 1965 & 1980 & 1995\end{array}$

h)
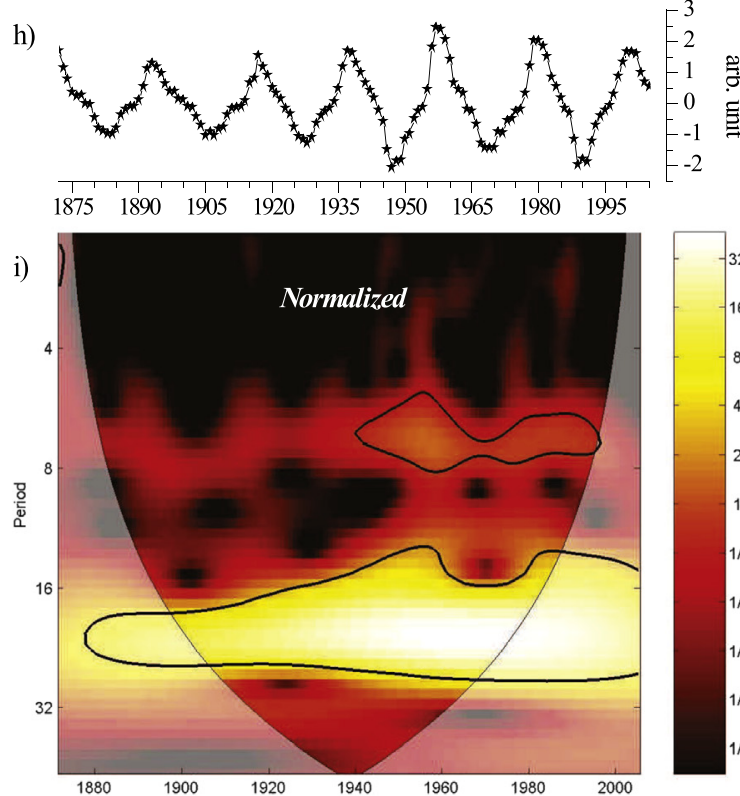

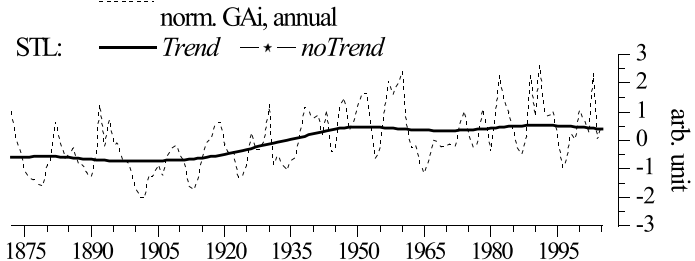

e)

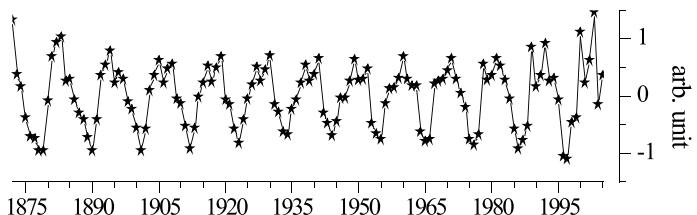

f)

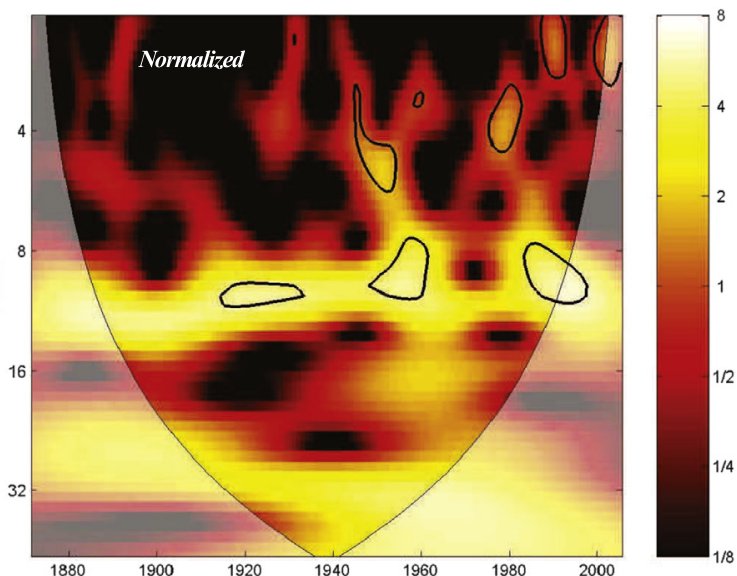

j)

$\mathrm{CO}_{2}$

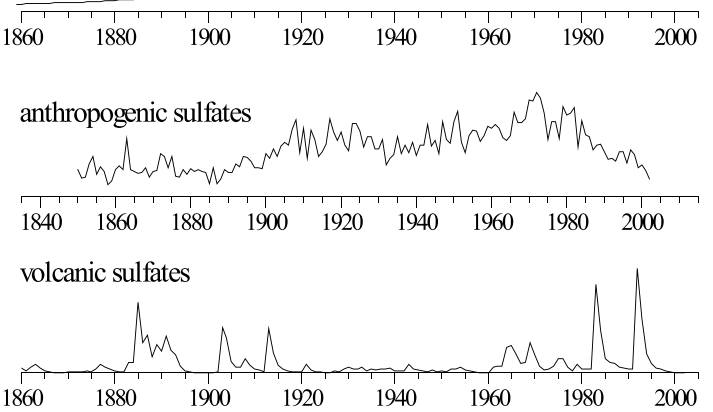

SSN

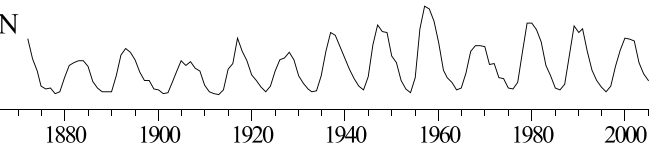

GAi
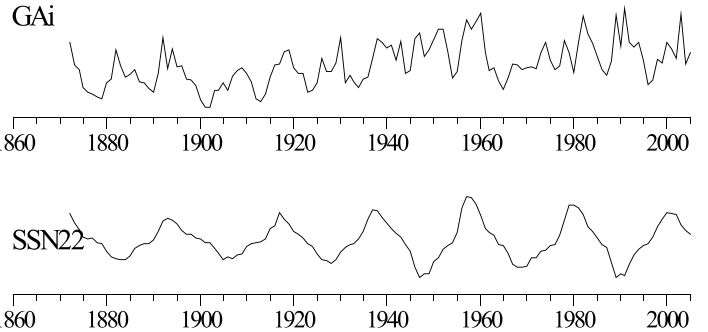

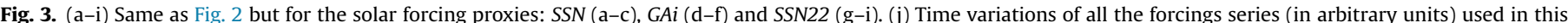

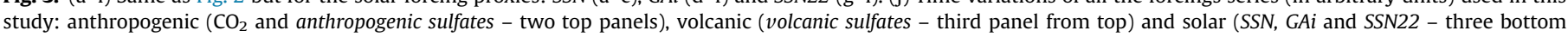
panels). 
wavelet coherence and regression analyses; however, all four series of the geomagnetic indices ( $a a, I H V, I D V, G A i)$ were used in the correlation analysis (see Section 3.2).

The 11-yr cycle of the solar activity results from the twice-long cycle of the variations of the solar magnetic field. During this 22-yr cycle there is an exchange of the energy between the poloidal and toroidal components of the solar magnetic field (SMF) (Hurrell, 2012). These two components can be associated with the magnetic fields observed in the polar and sunspot regions, respectively. The time variations of these two components of SMF show strong 22yr cycles that are shifted from each other by about half of the 11-yr cycle (the energy transition from the toroidal to the poloidal component starts at the declining phase of a 11-yr cycle, Hurrell, 2012). SMF, in turn, affect interplanetary magnetic field (IMF), solar wind conditions, geomagnetic activity and particle fluxes at the Earth's orbit. Therefore, observations of SMF and its polar (poloidal) component (solar polar magnetic field, SPMF) can be considered as physical parameters with a 22-yr cycle which through the IMF, solar wind (including the streams from the coronal holes), geomagnetic disturbances, particle fluxes etc. (Cliver et al., 1996; Lockwood, 2001; Hurrell, 2012) affect the Earth's environment.

Unfortunately, the observations of the SMF started only in the last quarter of the 20th c., e.g. by the Wilcox Solar Observatory (WSO). The WSO Polar Field series starts only in 1976 and can't be used for our studies. On the other side, since the sunspot 11-yr cycle results from the solar magnetic $22-\mathrm{yr}$ cycle, we can try to produce a proxy for the SPMF series using the SSN series with a sign. This proxy series (SSN22) can be created by applying a sign ( + or - ) to the SSN values in accordance (for example) with the polarity of the magnetic field of the leading sunspot in the northern solar hemisphere during the current 11-yr cycle (see e.g. Mursula et al., 2001) - see Fig. 3g. When compared to the SPMF series (solar northern hemisphere), this artificial SSN22 series shows strong anti-correlation $(-0.91, p$ values $<0.01)$ if the SSN22 series leads by $4-5$ years. This time lag is to be expected due to time evolution of the SMF components mentioned above (please, also, see Fig. S1f of the Supplementary Material for the comparison of the SPMF and SSN22 series, original and lagged forward). Therefore, the artificial SSN22 series can be considered as a proxy for the physical parameter - solar polar magnetic field variations if the time shift (SSN22 series leads by $4-5$ years) is taken into account.

Fig. 3 j represents the time variations of all the forcings series (in arbitrary units) used in our study: anthropogenic $\left(\mathrm{CO}_{2}\right.$ and anthropogenic sulfates - two top panels), volcanic (volcanic sulfates - third panel from top) and solar (SSN, GAi and SSN22 - three bottom panels).

\section{Methods}

\subsection{Data pre-processing}

The most of the series used in this study can be considered as consisted of a long-term trend and a detrended component. The last one can be divided into a cyclic (varying with some characteristic period $n p$ ) and residual components. To extract these components we used a decomposition method called STL or the Seasonal-Trend decomposition based on LOESS (LOcally wEighted regreSSion). This method allows one to decompose a series $X(t)$ into three additive components: a long-term trend $T(t)$, a cyclic component $C_{n p}(t)$ with predefined period $n p$ (in our case it is measured in years) and residuals $R(t)$ :

$X(t)=T(t)+C_{n p}(t)+R(t)$

The Cyclic and Residual components can be summed up into the noTrend components ("detrended" series). The STL decomposition is described in full details in (Cleveland and Devlin, 1988; Cleveland et al., 1990) and a brief summary can be found in the Appendices $\mathrm{A}$ and $\mathrm{B}$.

The STL procedure can be viewed as a filter that distributes the variations with different periods into three "channels". Those with periods close to predefined $n p$ value are included in the Cyclic component. The variations with periods greater than $n p$ are filtered into the Trend component. The rest (with periods shorter than $n p$ ) goes to the Residuals. This means that the choice of the $n p$ values is defined by the filtering purpose. In this study we used the STL with different $n p$ values to achieve two goals. The first goal is to smooth the original series removing short-term variations (with characteristic periods of 1-3 years). The second goal is to filter the original (or smoothed) series and subtract periodic and/or longterm variations or detrend the series.

The choice of the $n p$ value is defined by the characteristic period one wants to extract. Therefore, we used $n p=1$ to smooth series with significant short-term (period of 2-3 yrs) variations, and $n p=11$ and 22 were used for SSN and GAi, and SSN22 series, correspondingly. When the characteristic period is unknown $a$ priori or disputable the appropriate $n p$ value can be found in the analysis of the relations between the three components for different $n p$ values. It seems logical to try to increase the amount of variations (of the original series) that goes into the Cyclic component (or Trend component, depending on filtering purposes) and decrease the amount of the variations that goes into the Residuals. Some statistical parameters can be used to fulfill this task: (e.g.) standard deviation or correlation/congruence coefficients. We used this method to estimate the $n p$ values for the temperature and both sulfates series. For the temperature the "best" $n p$ values were in range of 20-26 (therefore, $n p=11$ and 22 were used) and for other series $n p=15$ was chosen. See also Table 1 for the list of $n p$ values and corresponding series. The STL components of the annual series for all parameters are shown in Figs. 1-3 (see Figs. S2-S5 from the Supp. Material for the corresponding components of the seasonal temperature series). To ensure that the STL

Table 1

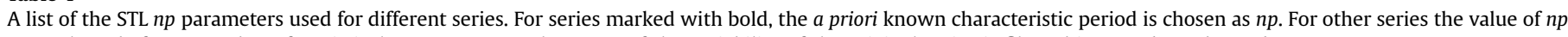
was selected after a number of statistical tests to ensure that most of the variability of the original series is filtered into Cyclic and Trend components.

\begin{tabular}{|c|c|c|c|c|}
\hline$n p$ & Filtered periods, yrs & Applied to series of... & Resulting series & Decomposition goal \\
\hline 1 & $1-3$ & Temperature, anthropogenic sulfates & Smoothed & $\begin{array}{l}\text { Smoothing procedure to remove short-term year-to-year } \\
\text { variations }\end{array}$ \\
\hline 11 & $10-12$ & SSN, GAi, Smoothed temperature series & Trend, Cyclic, Residuals, & Decomposition into two components: \\
\hline 15 & $13-17$ & $\begin{array}{l}\text { Volcanic sulfates, Smoothed anthropogenic } \\
\text { sulfates }\end{array}$ & noTrend & $\begin{array}{l}\text { (1) Cyclic with a characteristic period or "detrended", } \\
\text { (2) Long-term trend }\end{array}$ \\
\hline 22 & $20-24$ & SSN22, Smoothed temperature series & & \\
\hline
\end{tabular}


decomposition does not create false variabilities but only clarify and enhance those that already exist, we tested the decomposed components using the wavelet analysis (see Section 3.2). The examples of such analysis can be found in the Supp. Material, Figs. S6-S9.

\subsection{Data analysis}

\subsubsection{Wavelet analysis.}

The wavelet analysis was used to inspect the evolution of periodicities existing in a data set at different times. The wavelet cross coherence and phase technique was applied to analyze the coherence of two data sets, its evolution and the corresponding phase lag between the data sets. The results are visualized as timefrequency spectra where the powers are represented by different colors (corresponding color map is shown nearby each spectrum). The statistical significance of the computed powers is calculated against the red-noise background. Statistically significant zones of the spectrum (we use the 95\% significance level) are contoured by black lines. An influence of boundary effects is taken into account and one should trust only the results inside the so-called "cone of influence". On the wavelet coherence plots the phase relation between the two analyzed data sets - phase lags - are visualized by arrows. If an arrow is directed from left to right then the data sets are in phase, if from right to left - in anti-phase, if from top to bottom - the first data set leads the second one in quarter of corresponding period. The detailed description of these methods can be found, for example, in (Torrence and Compo, 1998; Maraun and Kurths, 2004).

\subsubsection{Correlation and multiple regression analysis.}

The pairwise correlation coefficients $(r)$ and the pairwise running correlation coefficients (using a 20 years long window) between the temperature and the forcing series were calculated for the whole period from 1888 to 2001. Significances of these $r$ for singular and multiple comparisons were calculated using the nonparametric Monte-Carlo approach when during each of 10,000 runs the artificial series were constructed using (1) the "phase randomization procedure" which consists of the direct FFT followed by the inverse FFT with the same power spectrum but random phase (Ebisuzaki, 1997) or (2) the "bootstrapping with moving blocks" randomization procedure (e.g. Künsch, 1989; Lahiri, 1999). Each time the randomization procedure creates an artificial series with the same autocorrelation and the effective degree of freedom as the original one. The artificial series is subjected to the same type of the correlation analysis as the original series. Singular comparison significance ( $p$ value) takes into account only the probability of a random series to have the same (or bigger) absolute value of $r$ as in the case of a specific pair of the original series. Multiple comparisons significance (meta $p$ values) takes into account the same probability but for the whole sequence of pairs of the original series.

The combined influence of different forcings on the temperature variations was studied using linear multiple regression models (MRMs). A "best subset" technique was chosen to ensure that only those regressors (forcings) that are most significant and most influential for the current model were selected. The "best subset" was estimated using a so-called adjusted squared coefficient of multiple determination $\left(\mathrm{R}_{\mathrm{adj}}{ }^{2}\right)$. The value $\mathrm{R}_{\mathrm{adj}}{ }^{2} \times 100 \%$ shows the percent of the variations of the dependent parameter (temperature) explained by the current MRM. While MRM is not a proof for cause-and-effect relationships between the dependent variable and regressors, it gives a quantification of the relationships between the dependent and independent variables on the base of their similarities (Gámiz-Fortis et al., 2011). Four types of MRMs listed in the Table 2 were calculated consecutively by
Table 2

Forcings included in multiple regression models (MRMs): anthropogenic (model \#1 and model \#2), anthropogenic and volcanic (model \#3), anthropogenic, volcanic and solar (models \#4). The CO2 forcing was excluded from the MRMs of the noTrend temperature components (see Section 4.2).

\begin{tabular}{llccc}
\hline Forcing/natural variability & \multicolumn{4}{l}{ Multiple regression model (MRM) } \\
\cline { 2 - 5 } & $\# 1$ & $\# 2$ & $\# 3$ & $\# 4$ \\
\cline { 2 - 5 } & + & + & + & + \\
$\mathrm{CO}_{2}$ & & + & + & + \\
Anthropogenic sulfates & & & + & + \\
Volcanic sulfates & & & & + \\
SSN22 and SSN or GAi & & & & + \\
\hline
\end{tabular}

adding one of the forcing parameters after another.

\section{Results and discussion}

\subsection{Brief description of observed temperature variations}

Figs. 1a-3a shows the original, Smoothed and Trend components of the variations of the annual temperature and forcings series. For the temperature the 11-yr Cyclic and 22-yr Cyclic STL components are shown - see Fig. $1 \mathrm{~b}, \mathrm{e}, \mathrm{h}$ and k. For the forcings series the noTrend STL components are show - see Fig. $2 \mathrm{~b}$ and e and $3 \mathrm{~b}$, e and $\mathrm{h}$. The most prominent feature observed in the temperature variations from 1888 to 2001 is the tendency to the gradual growth of Tmin and Tmax (Fig. 1a and d - annual series). This growth is clearly seen in the Tmin annual series, in the winter series of both Tmin and Tmax, and in all other Tmin seasonal series (please see Supp. Material, Fig. S2). These variations result in a gradual growth of the annual (Fig. 1g), winter, summer and autumn (Supp. Material, Fig. S4) averT values. On the other hand, the rate of the growth of Tmin and Tmax are different during the first and the second halves of the $20^{\text {th }} \mathrm{c}$. This difference leads to variations of DTR consisting of a growth before about 1950s-1960s and a decrease afterwards (Fig. 1e - annual series and Fig. S5 in the Supp. Material for the seasonal series).

Comparing to other analysis available for the Iberian Peninsula (e.g. del Rio et al. 2012; Gómez-Navarro et al., 2010, 2012, 2013; Luterbacher et al., 2004; Rozas, García-González, 2012) the data from Portuguese stations show similar trends for Tmin, Tmax and averT but there are differences in the DTR variations. For example, simulations and data analysis (e.g. del Rio et al. 2012; GómezNavarro et al., 2010) for the Continental Spain stations demonstrate stronger growth of Tmax than Tmin. This results in the growing trend of DTR during the 2nd half of the 20th c. This difference between the Spanish and Portuguese data can be caused (e.g.) by the coastal position of Portugal and a stronger influence of the Atlantic Ocean and NAO. del Rio et al. (2012, see also references therein) argue that the circulation pattern variations can affect the temperature regime of the Iberian Peninsula not only directly through the advection of the warm/cold air masses but through the changes of the cloud cover and, consequently, the amount of the incoming solar radiation. This type of the relations between NAO and Iberian temperatures was found also by Sánchez-Lorenzo et al., 2007, 2012. Finally, the DTR trends are different over the Europe: here there are regions with a uniform DTR trend and regions with a growth-decrease type of variations (Durre, Wallace, 2001; Heino et al., 1999; Karl et al., 1993; Liu et al., 2002; Luterbacher et al., 2004; Makowski et al., 2008; PAGES 2k Consortium, 2013; Weber et al., 1994; ). These DTR variations are often associated with the changes in the cloud cover and following dimming or brightening - decrease or increase of the solar radiations reaching the ground (del Rio et al. 2012). 
a)
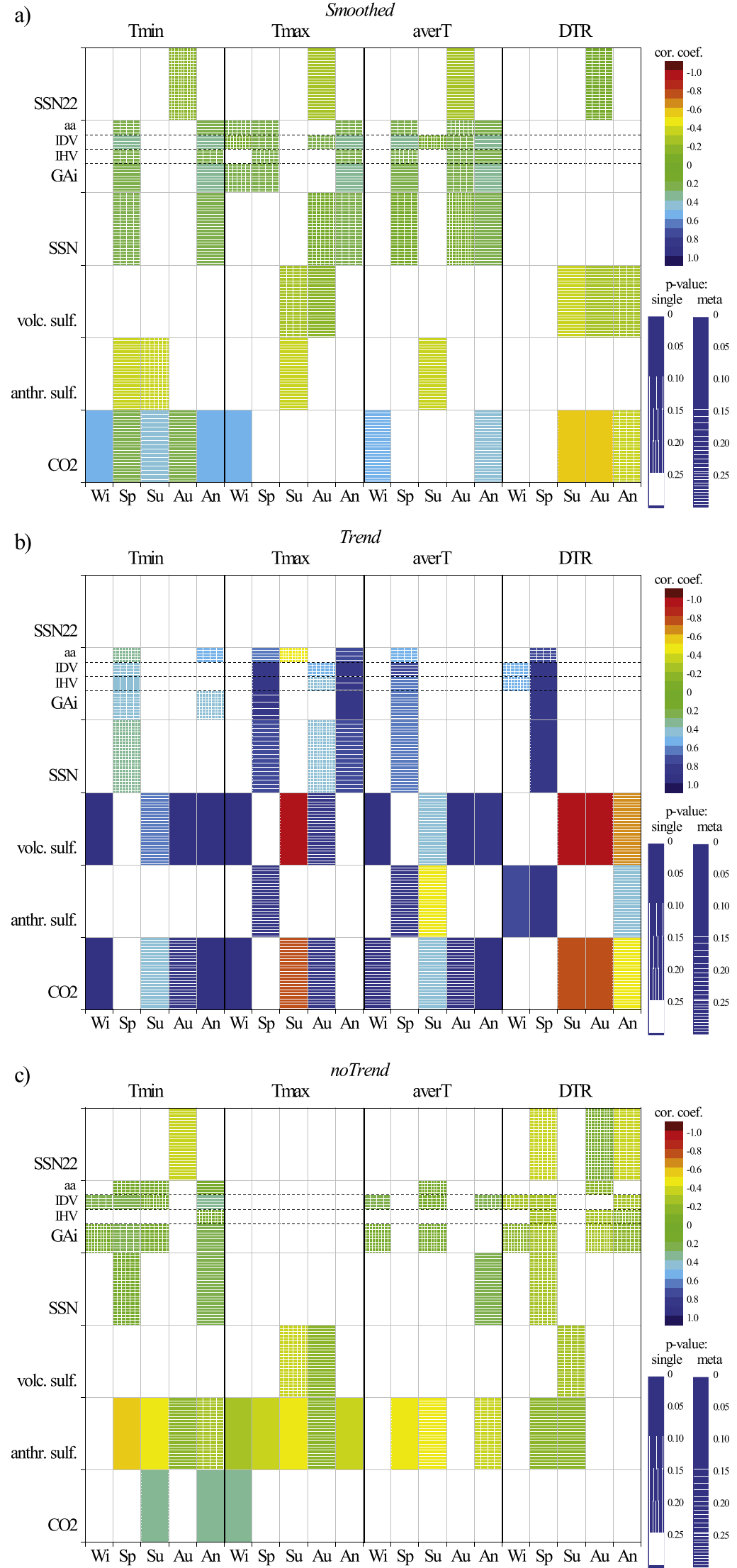

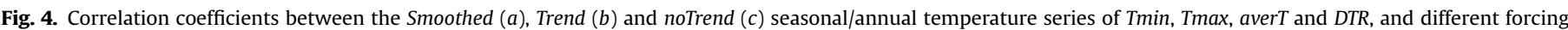

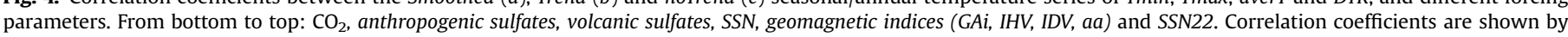

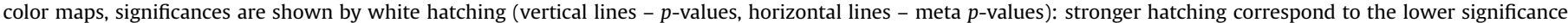
level. 


\subsubsection{Wavelet analysis}

The most interesting result of the wavelet analysis of the temperature series (see Fig. 1c, f, i and 1 for the Smoothed annual series and Supp. Material, Figs. S2-S5, panels c, f, i and 1, for the Smoothed seasonal series) is the appearance of significant (with at least 95\% significance level) decadal and bi-decadal oscillations. Most of the Smoothed temperature series demonstrate these oscillations in the middle of 20th c. (from ca. 1930 to ca. 1960). The strongest decadal signal is observed in the DTR data, whereas the strongest bi-decadal signal is obtained for the averT data. In the wavelet spectra of some series the two separate islands of decadal and bi-decadal oscillations are merged into one big band (as, e.g., is shown in Fig. $1 \mathrm{f}$ and $\mathrm{l}$ for the Smoothed annual series of Tmax and $D T R$, correspondingly). Seasonal data demonstrate also some higher frequency variations visualized in the wavelet spectra as significant but unstable islands. The existence of the multi-decadal signal in the temperature variations is also supported by the STL decomposition with the $n p$ values of 11 and 22 (Fig. $1 \mathrm{~b}, \mathrm{e}, \mathrm{h}, \mathrm{k}$ ). As one can see, for some of the temperature series a strong Cyclic component with a period of 11 (22) years can be decomposed (as, e.g., in case of 11-yr Cyclic component for Tmin, Tmax and DTR and in case of 22-yr Cyclic component for Tmax, averT and DTR) but for others the corresponding component shows no significant oscillations (as, e.g., in case of 11-yr Cyclic component for averT and in case of 22-yr Cyclic component for Tmin).

The results of the wavelet analysis of the forcings' parameters
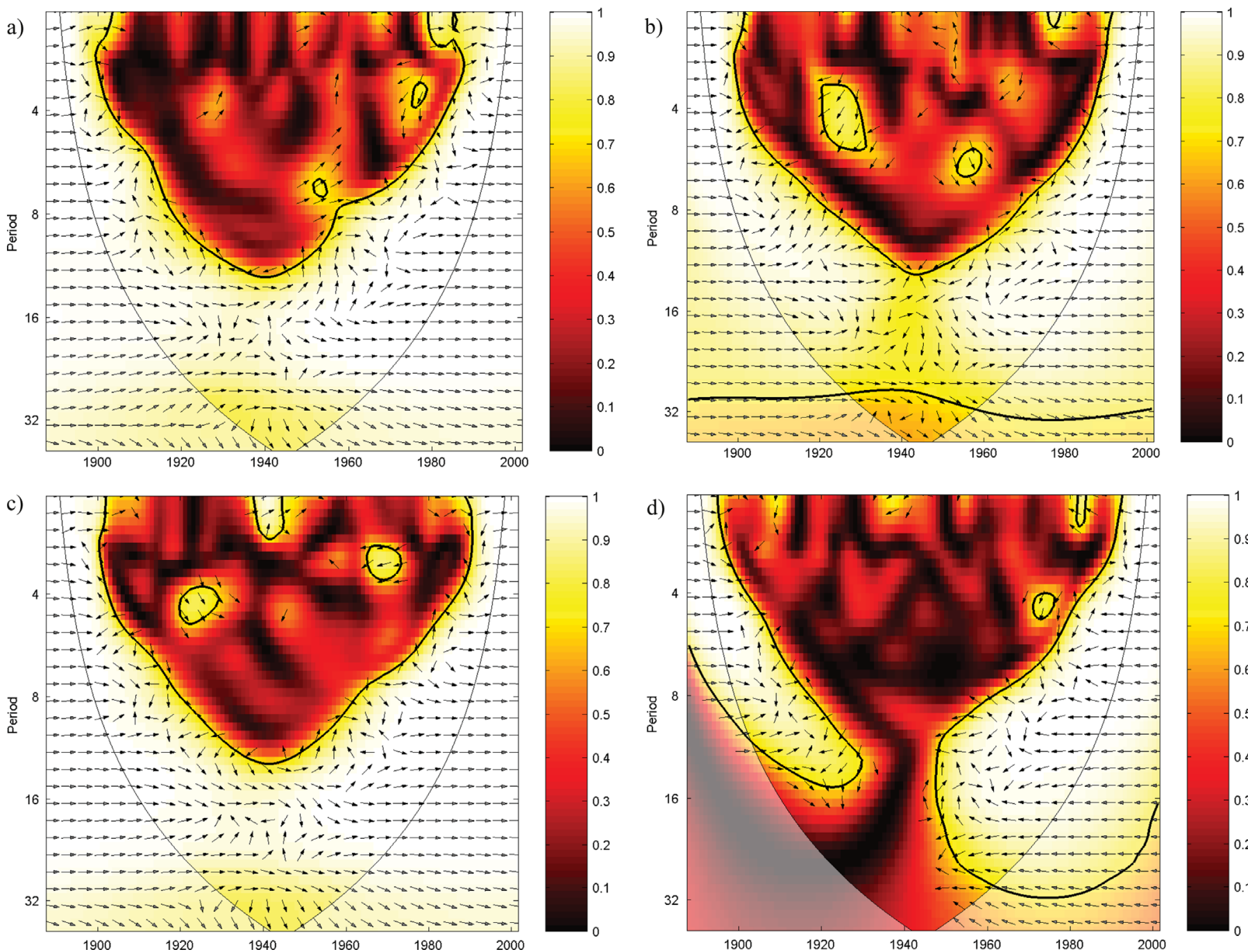
pogenic sulfates (Fig. $2 \mathrm{a}-\mathrm{c}$ ) detected by the wavelet analysis is not clear. It could be an artifact of data collecting procedures but, on the other hand, these oscillations could reflect variations of the atmospheric circulation. The oscillation patterns were also found in the wavelet spectra of the volcanic sulfates series (Fig. 2d-f). However, they are artifacts of the method: volcanic eruptions are independent to each other, so any of the detected quasi-periodicities occur by chance. As expected, the wavelet spectra of the SSN and the SSN22 series (Fig. 3c and i, correspondingly) show strong and statistically significant variations with $11-\mathrm{yr}$ and 22 -yr periods, respectively, while the 11-yr GAi variations (Fig. 3f) are less prominent and their significance is unstable due to the significant changes in the amplitude and length of the geomagnetic cycles (Fig. 3d, dashed line and 3e).

\subsection{Effect of anthropogenic and volcanic forcings seen by different methods}

The strong correlation of the $\mathrm{CO}_{2}$ forcing and the temperature variations is seen in Smoothed (Fig. 4a) and Trend (Fig. 4b) temperature series. The response of the Tmin series is stronger and the statistical significances of the correlation coefficients are higher both during the cold (winter-early spring) and the summer seasons. The Tmax, and therefore averT, series show much weaker correlation with the $\mathrm{CO}_{2}$ increase, which is nevertheless very

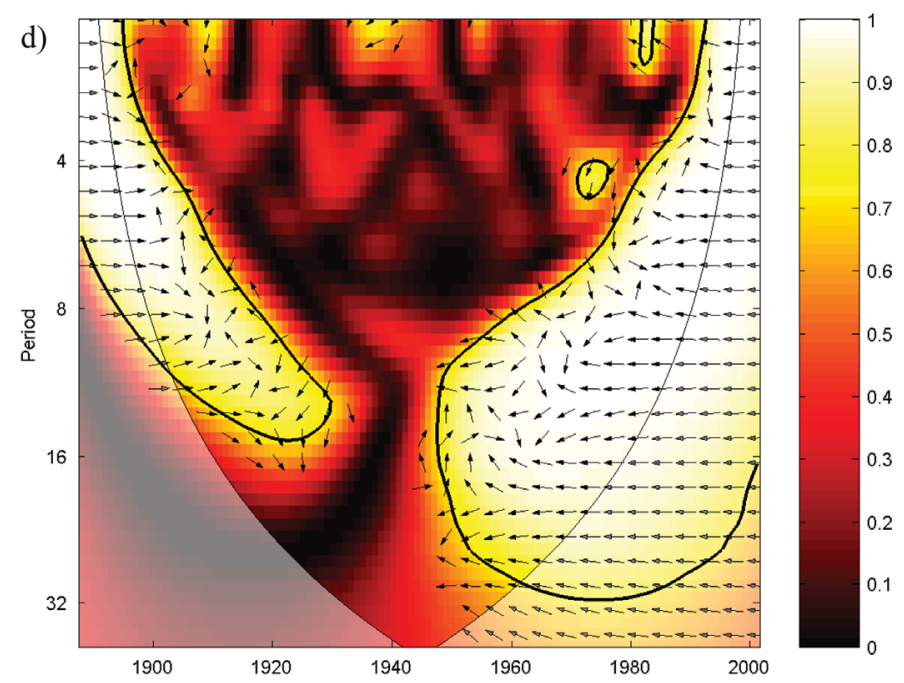

Fig. 5. Wavelet coherence between the $\mathrm{CO}_{2}$ variations and Trend components of annual temperature parameters: Tmin - $a$, Tmax - $b$, averT - $c$, DTR - $d$. 
strong in the cold period of a year. Consequently, there is an anticorrelation between the DTR and the $\mathrm{CO}_{2}$ variations for the summer-autumn period. Among all the noTrend components (Fig. 4c) of the temperature series only the Tmin annual and summer series and Tmax winter series show the statistically significant response to the $\mathrm{CO}_{2}$ growth. We interpret this as an indication that the $\mathrm{CO}_{2}$ forcing affects mostly the long-term temperature Trends leading to the gradual warming (mostly during the cold period of a year). These results of the correlations analysis are confirmed by the wavelet coherence analysis (Fig. 5). There is a strong in-phase signals in the coherence spectra between the annual series of $\mathrm{CO}_{2}$ and the Trend components of Tmin, Tmax and averT (Fig. 5a, b and c, correspondingly) as well as change of the power and phase in the coherence spectra between $\mathrm{CO}_{2}$ and the Trend component of the DTR series (Fig. 5d). Based on these results, we included the $\mathrm{CO}_{2}$ forcing in the regression analysis (discussed below) of the Smoothed and Trend temperatures components and excluded it from the MRMs of the noTrend components as non-important for the temperature variations on the corresponding time scales.

The correlation analysis shows a weak but statistically significant anti-correlation between the temperature series and the series of the anthropogenic sulfates, especially for the warmer seasons (see Fig. 4a and c). This anti-correlation is most prominent when detrended series (noTrend) are considered. The wavelet coherence analysis (Fig. 6a - annual noTrend averT, and Fig. 6c -
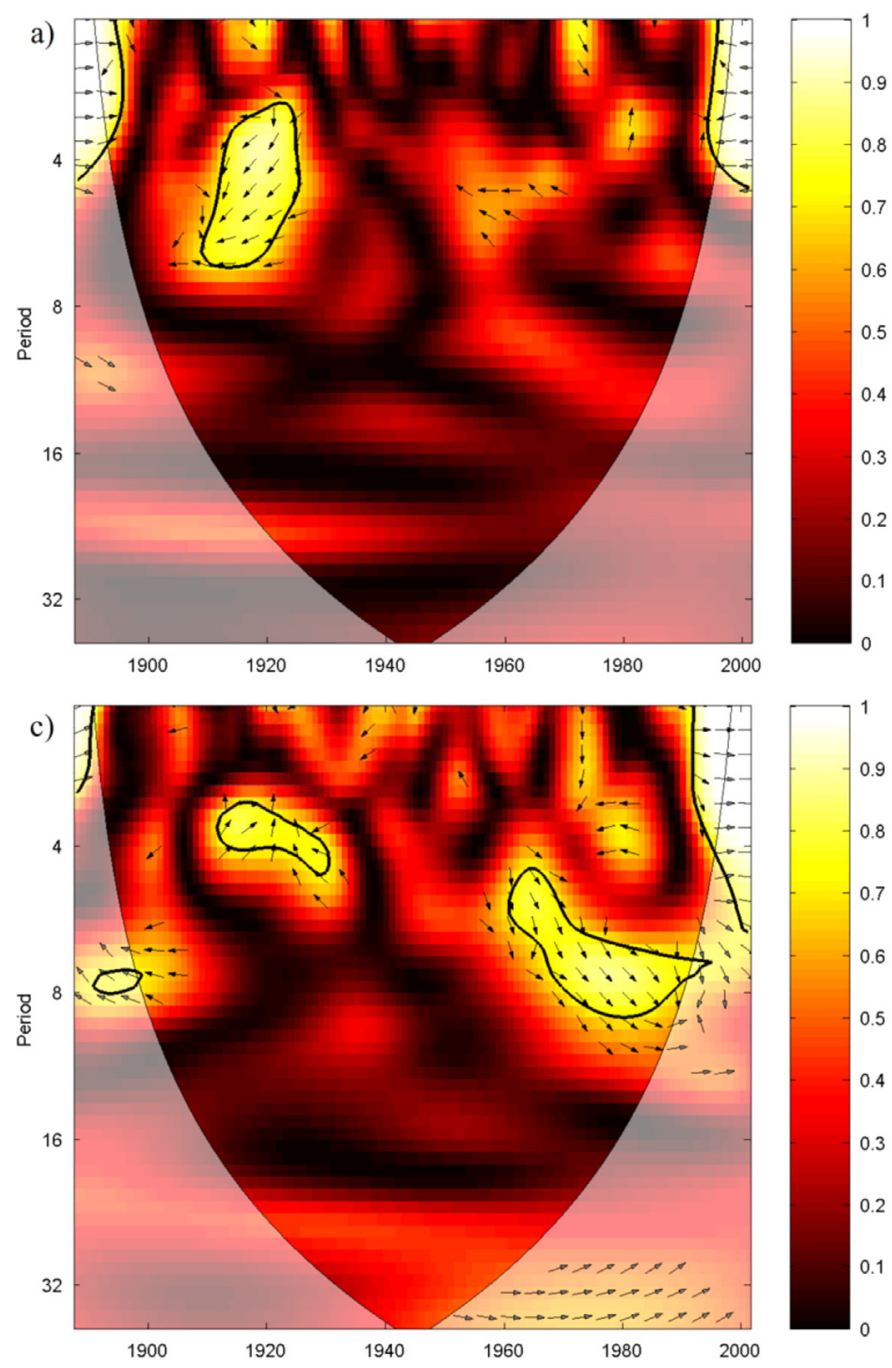

annual noTrend DTR) confirms this conclusion. The wavelet coherence spectrum between the noTrend averT and anthropogenic sulfates series have one statistically significant island $(\sim 1900$ 1920) of the coherent signal (signals are in anti-phase) and the similar spectrum for DTR series shows two such islands ( $\sim 1910$ 1930 and $\sim 1960-1990$ ) of anti-phase signals.

The detection of the effect of the volcanic forcings is more difficult with the time resolution and time scale of our temperature series. However, the wavelet coherence analysis allows us to detect statistically significant signal: the anti-correlation between the temperature and volcanic sulfates series (see Fig. $6 \mathrm{c}$ and d for the coherence spectra for the noTrend averT and DTR series, correspondingly). The detected volcanic signal is clearly seen in the averT wavelet coherence spectrum: there are two islands of the significant anti-phase signals during two epochs of strong volcanic eruptions: 1890-1920 and 1980-1990. In the DTR coherence spectrum the volcanic signal is seen but not statistically significant. However, there is a significant anti-phase signal around the period of about 30 -yr. This signal is, most probably, originates from a roughly 30-yr time intervals between the strongest volcanic eruptions in the 19th-20th c.: $\sim 1860$, $\sim 1890, \sim 1920, \sim 1965, \sim 1980-90$ (see Fig. 2d-f). The correlation analysis also detects the anti-correlation between the variations of the volcanic sulfates and temperature series; however the correlations coefficients are weak and statistically significant only for a number of series (Fig. 4a and c).

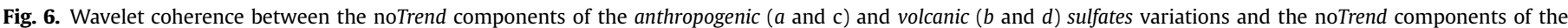
annual series of averT ( $\mathrm{a}$ and $\mathrm{b}$ ) and DTR ( $\mathrm{c}$ and $\mathrm{d}$ ). 

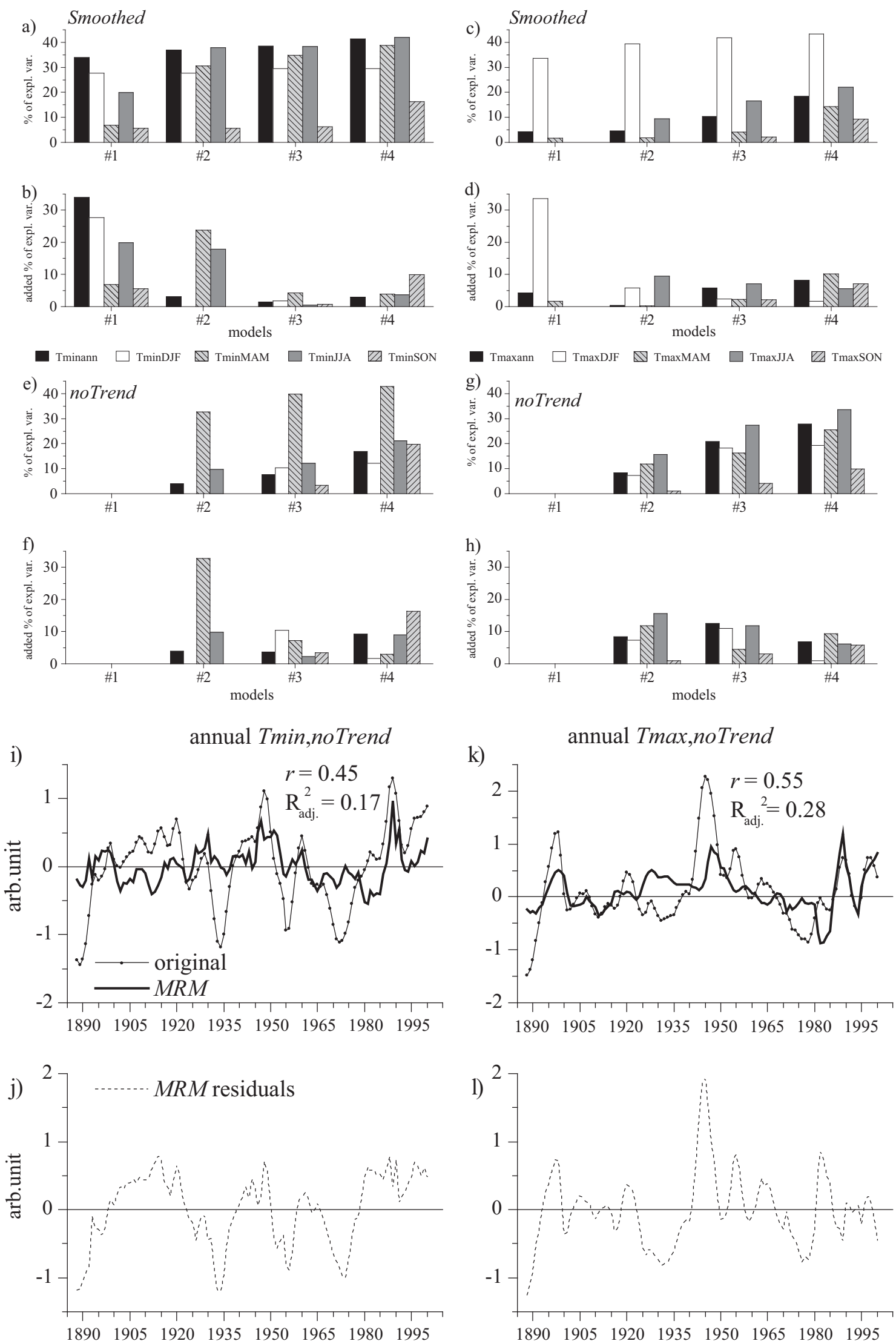

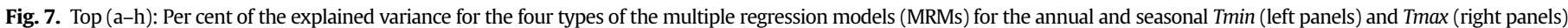

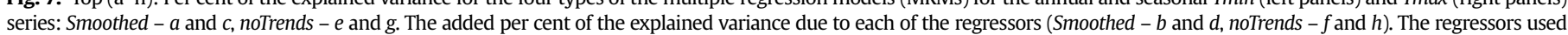

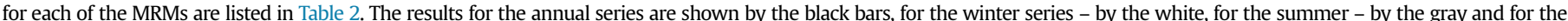

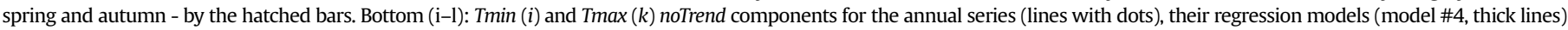
and residuals $(\operatorname{Tmin}-j, \operatorname{Tmax}-l)$. Correlation coefficients $(r)$ between the original and the predicted series as well as the explained variance $\left(R_{a d j .}{ }^{2}\right)$ are shown. 

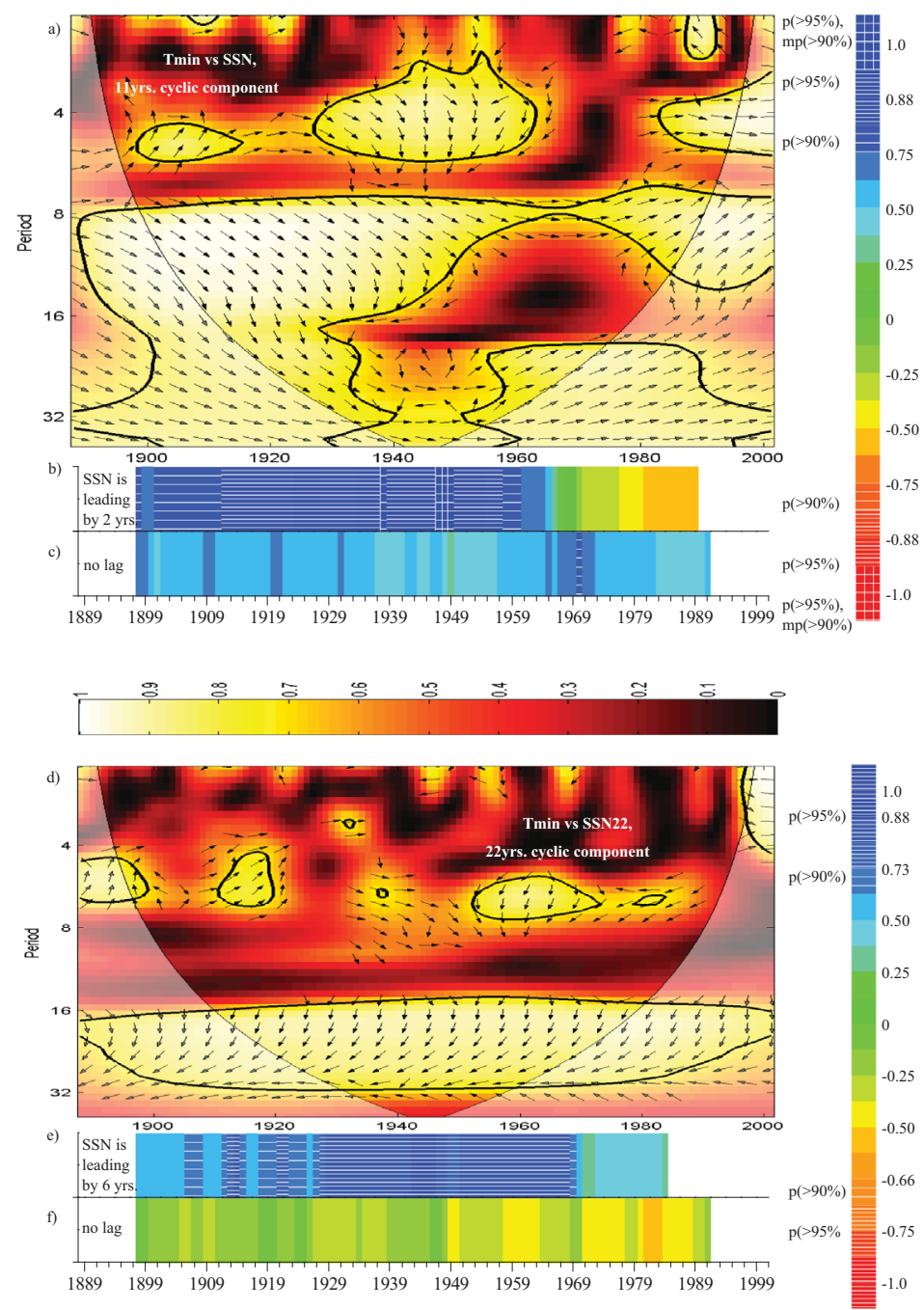
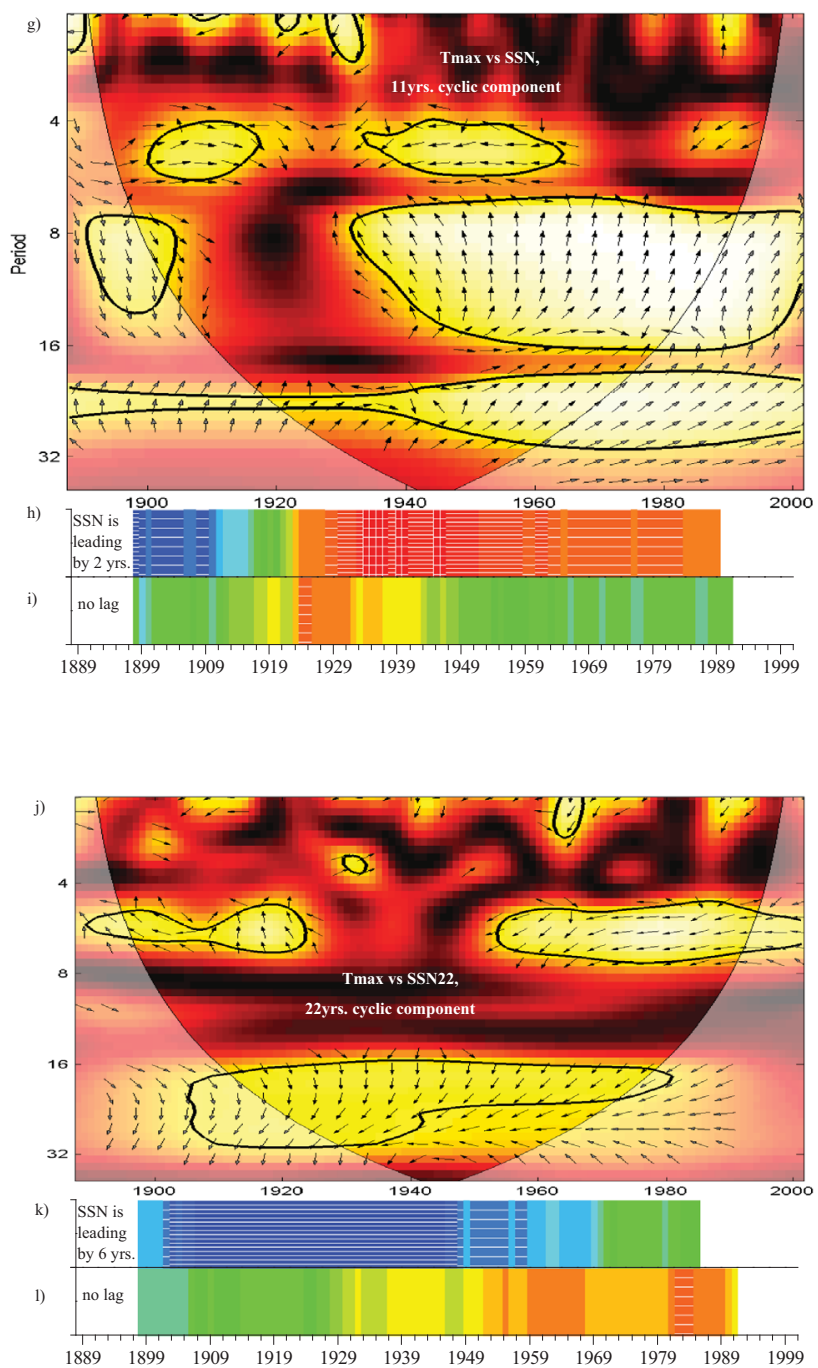

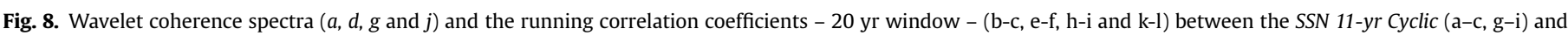

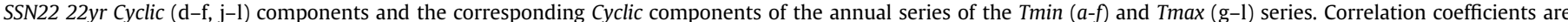

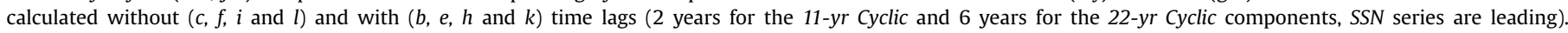

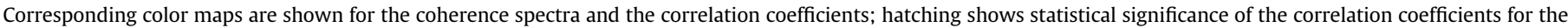
pairwise $(p)$ and multiple $(m p)$ comparisons.

The results of the correlation and wavelet analyses discussed above are confirmed by the multiple regressions models that were constructed to explain seasonal and annual temperature variations using forcings series as regressors. The results of such modeling are shown in Fig. 7 for the Tmin and Tmax series. Panels (a-d) show per cent of the explained variance of the corresponding temperature variations by the selected set of regressors (MRM models \#1-\#4) and panels (e-h) show the individual input of each of the forcings. Over all, there were constructed four types of the MRM models. The list of the regressors for each of the types is shown in Table 2. These models were constructed for Smoothed, Trend and noTrend seasonal and annual temperature series. As was mentioned above, the $\mathrm{CO}_{2}$ forcing was excluded from the analysis of the noTrend temperature series.

For the Smoothed series (Fig. 7a-d) the MRMs (models \#1-\#3) constructed for the seasonal and annual series succeed to explain up to $44 \%$ of the Tmin variations and up to $35 \%$ of the Tmax variations. Fig. 7a and $\mathrm{c}$ show the percent of the explained variance of the temperature series of Tmin and Tmax, correspondingly, and Fig. $7 \mathrm{~b}$ and $\mathrm{d}$ show the contribution of different forcings in the terms of the added explained variance due to the use of a new regressor comparing to the previous type of model. Fig. 7e-h shows same results for the noTrend temperature series. The MRMs for the Tmin series are more successful in the reconstruction of the temperature variations than the MRMs for the Tmax, and only in case of the winter Tmax series (white bars in Fig. 7) the explained variance is comparable with the one of the Tmin models. $\mathrm{CO}_{2}$ has the strongest contribution as a regressor to the variations of the annual and winter Smoothed Tmin and the winter Smoothed Tmax series (model \#1). It explains up to about $35 \%$ of the total variance of the original series. $\mathrm{CO}_{2}$ is followed by the anthropogenic sulfates (model \#2 - up to $20-25 \%$ of the total variance). The volcanic sulfates (model \#3) explain up to $10 \%$ of the total variance of the Smoothed temperature series.

The multiple regression models constructed for the Trend components (not shown here) explain greater part of the temperature variation than the MRMs for Smoothed components. The larger response is partly due to the smoothness of the Trend series and consequent decrease of the effective degree of freedom. The variations of the annual and seasonal Trend series of the Tmin (except summer) and the Tmax (except spring) can be explained by the $\mathrm{CO}_{2}$ growth. Addition of the Trend components of the anthropogenic and volcanic sulfates improves the quality of the MRMs for spring, summer and autumn temperature series. 
Similar results were obtained using the multiple regression models for the noTrend components (Fig. 7e-h). The prediction quality of the noTrend models is shown in Fig. 7e and $\mathrm{g}$ for the Tmin and Tmax series, respectively. The anthropogenic sulfate noTrend component (model \#2) is the most influential regressor for the spring Tmin and both sulfates series are important regressors for the Tmax noTrend series. Concerning the MRMs (model \#3 and \#4 - see Section 4.3) for the seasonal temperature series, one can see that both for the Tmin and Tmax series our MRMs (based only on the external forcings) for the autumn-winter season explain smaller part of the temperature variations (up to 11-21\%) than such models for the spring-summer season (up to 26-44\%). This is in an agreement with the role played by the atmospheric circulation in the variations of the Portuguese (coastal) climate during different seasons. During the cold part of a year this region is under the strong influence of the Atlantic cyclones, and, therefore, significant part of the temperature variations has to be caused by variations of NAO and other circulation patterns in the region.

\subsection{Decadal and bi-decadal temperature variations and the role of the solar forcing}

As was mentioned in Section 4.1, the temperature series show decadal and bi-decadal variations. These (bi-)decadal signals can be extracted both by the STL decomposition (see Fig. 1b, e, h, k) and the wavelet analysis (see Fig. 8). The statistically significant (bi-)decadal signals appear in the temperature wavelet spectra (Fig. 1c, f, I, 1) mostly between ca. 1930 and ca. 1990. The main sources that can be responsible for the temperature variations with quasi-(bi-)decadal periods are solar external forcing and internal variability of the atmosphere and atmosphere-ocean systems. The mathematical methods used in our study do not allow us to reach a final verdict on what kind of source is responsible for the (bi-)decadal variations observed in the Portuguese temperature series. However, some conclusion can be drawn.

First we discuss the results of the correlation, multiple regression and wavelet coherence analyses of the relations between the temperature series and series used by us as proxies for the solar forcing: SSN, GAi and SSN22 (which is a proxy for the solar polar magnetic field variations with a lag of 4-5 yrs, see Section 2.2.3). The wavelet coherence analysis of the 11-yr (22-yr) Cyclic components of the Tmin and Tmax vs. SSN, GAi (SSN22) series shows strong and statistically significant coherent signals at these periods (see Fig. 8 for annual series, and Figs. S13-S16 in the Supp. Material for the seasonal series). These signals are visible in the coherence spectra during almost the whole studied time interval but the phase changes with time. Accordingly to the wavelet coherence spectra there are time lags between the temperature and the solar cycles of about $1 / 8-1 / 4$ of the corresponding periods (sunspot series lead). These lags are confirmed by the running correlation analysis (see Fig. 8b-c, e-f, h-i and k-l). The running correlation coefficient calculated without lag shows very weak, if any, correlations between the temperature and the solar Cyclic components. On contrary, in the cases when the solar series lead the temperature series by 1-2 (11-yr Cyclic components) or 6 (22yr Cyclic components) years the correlation is strong and statistically significant. The running correlation analysis also confirms the phase shifts in the wavelet coherence spectra of the temperature and the SSN series that took places around 1910 and around 1930. The MRMs constructed for the Tmin and Tmax series using full set of the forcings parameters (model \#4, see Table 2) have bigger predictive power than the regression models based only on the anthropogenic and volcanic forcings (model \#3) - see Fig. 7. The addition of the solar and geomagnetic indices to the MRM improves the explained variance for the Smoothed Tmax and Tmin models for spring and autumn series (explain extra $7-10 \%$ of the variance, Fig. 7a-d) - the seasons of more frequent geomagnetic disturbances. The use of the Trend components of the solar and geomagnetic indices improves the model prediction level by $18 \%$ for the summer Trend Tmin series and by $16-17 \%$ for the spring and autumn Trend Tmax series (not shown). For the noTrend series the solar and geomagnetic indices are less influential regressors comparing to other forcings, except for the Tmin series for the summer and autumn. For these specific series the addition of the $S S N$ or GAi to the MRM increases the value of the explained variance by up to $15 \%$, which is comparable to the contribution of other regressors. Two examples of the MRMs are shown in Fig. 7i-1 for the annual Tmin and Tmax noTrend series (the corresponding MRM models for the seasonal Tmin and Tmax noTrend series can be found in the Supp. Material, Figs. S10-S11). The correlation coefficients between the original series and the models are quite high ( 0.45 for the Tmin and 0.55 for the Tmax series) and the explained variances $\left(\mathrm{R}_{\mathrm{adj}}{ }^{2} \times 100 \%\right)$ are $17 \%$ and $28 \%$, respectively.

Furthermore, it was found that in most cases the MRMs with the GAi as one of regressors have better prediction quality than MRMs with the SSN series as a regressor. However, we cannot say with certainty that the $G A i$ is a better regressor than the SSN. For example, the correlation analysis done using the SSN and GAi series (Fig. 4) shows that overall the correlation coefficients between the temperature and geomagnetic series are higher and more statistically significant then ones between the temperature and SSN series, however the statistical significance of such correlation coefficients is quite low. Besides, as in the case of the MRM, higher (and statistically more significant) correlations coefficients are obtained for the spring, autumn and annual temperature series. It has also to be mentioned that the results of the correlation analyses done using all four geomagnetic series (see Section 2.2.3) are consistent and for now we can not select one of the geomagnetic indices as most potential for the studies of the solar activity effect on Earth's climate.

The relations between the temperature and SSN22 series need special discussion. Both the wavelet coherence and the regression analyses (Fig. 8d-f and j-l) for the 1888-2001 time interval show that the correlation between the atmospheric and solar series increases when the solar series is shifted forward by $\sim 6 \mathrm{yr}$. However, as was mentioned in Section 2.2.3, there is a time lag of $\sim 4-5$ yrs between the SSN22 and SPMF series. The combination of these two facts results in a following hypothesis: an anti-correlation with a time lag of $\sim 1-2$ yrs is expected if the temperature 22Cyclic series are compared with the equivalent STL component of the SPMF series. We did the corresponding calculations and found that for the period of the SPMF observations that overlap with the temperature series (1977-2001) there is indeed the anti-correlation between the temperature and SPMF series with a lag of 1-2 yrs (SPMF leads). Unfortunately, this overlapping exists only during a very short time period ( 25 yrs or about one magnetic solar cycle), therefore the statistical significance of these correlation coefficients is very low. Nevertheless, it has to be noted, that the time lag length (1-2 yrs.) between the 22-yr cycles of SPMF and the atmospheric parameters is in consistence with the time lag obtained for the 11-yr cycles (as is shown in Fig. 8a-c and g-i). This allows us to assume that both the decadal and bi-decadal variabilities of the solar forcings affect the Earth's climate through the similar (or the same) mechanism that is driven by the changes of the solar magnetic field components (toroidal and poloidal which, to some extent, can be associated with the magnetic field of sunspots and solar polar regions, respectively) and resulting changes in the solar wind, magnetospheric and ionospheric conditions and variations of the fluxes of the energetic particles (galactic and solar cosmic rays, magnetospheric relativistic electrons etc.) - see (Lam and Tinsley, 2015 and Mironova et al., 2015) for reviews of such mechanisms. 
Multi-decadal signals are often found in the climatic series. For example, Meyers and Pagani, 2006 found quasi-25-yr periodicity in the variations of the temperature dependent tree-ring series and Lohmann et al., 2004 found multi-decadal variations of the sea surface temperature (SST) and sea level pressure (SLP) attributed to different solar cycles (Schwabe, Hale, and Gleissberg). However, despite the strong statistical significance of coherence spectra and correlation coefficients, our results cannot be considered as a definitive proof of the solar influence on the temperature variations. The first difficulty is the phase instability between the climatic and solar signals. This non-stationarity of the decadal signal in our temperature series is confirmed by the two independent methods correlation and wavelet coherence analyses. Please note that the values of the time lags are stable in time during these specific periods of the statistically significant co-variations of the temperature and solar parameters (e.g. from 1888 to about 1950 for the Tmin 11-yr Cyclic component as shown in Fig. 8a and from 1888 to 2001 of the for the Tmin 22-yr Cyclic component as shown in Fig. 8d). Similar phase shifts were already found in many other studies of the relations between the solar and climatic parameter: e.g., Gray et al., 2013 found such time lags in observed variations of the SST and SLP fields for the Northern Hemisphere. The climate variations are lagged behind the solar 11 -year cycle by $2-3$ years for SST (and by 3-4 years for SLP). Same type of behavior was also obtained in simulations. The mechanisms proposed to explain these lags are related to the stratospheric influence of the lower atmosphere, ocean-atmosphere interaction and variations of the NAO patterns. The analysis of the rain level for the Fortaleza Island (Brazil) by Gusev and Martin (2012) also shows phase change between the climatic and solar series around the middle of the 20th c.

Another reason to question the reliability of the strong solar forcing on the climatic series is the existence of internal (bi-) decadal modes in the atmosphere-ocean system or appearance of such periodic variations under the influence of other forcings. For example, (Reichler et al., 2012) in their analysis of the Atlantic Meridional Overturning Circulation (AMOC) found a pronounced peak in the spectrum of the simulated AMOC series at $\sim 20 \mathrm{yr}$ oscillation period when the atmospheric forcing was included in the oceanic model. Zanchettin et al. (2012, 2013) argue that strong volcanic eruptions (SVE) can be responsible for the appearances of the decadal/bi-decadal variations in the lower atmosphere seen both in the temperature and in the circulation patterns. They used reconstructions as well as climate models to analyze responses of the atmosphere and ocean to the SVE during the last millennium. The estimated length of the post-eruption effect is about a decade for the surface temperature and about 20-25 years for the oceanic circulation patterns. On the other hand, this means that even in case of a series of SVEs separated each from another by about decade, the quasi-decadal signal in the atmospheric temperature will fade after about 10-15 yrs after the last eruption (faster for the lower latitudes). This contradicts with our results. From the middle of 19th and until the end of the 20th c. there were two epochs of frequent and strong volcanic eruptions (Fig. $2 \mathrm{~d}-\mathrm{f}$ ): from 1860 s to 1920 s and from 1960s to 1990s). As one can see in Fig. 1 the decadal signals in the temperature series are strong and statistically significant only during the period of no SVE (from about 1920s to 1960s) and bi-decadal signal appears during almost all the studied period (except the last 20 years). The coherence spectra and the correlation coefficients between the Cyclic components of temperature and SSN and SSN22 (Fig. 8) also show no fading of the signals between these two epochs of frequent volcanic eruptions. Besides, the comparison of the wavelet coherence spectra shows that the coherent decadal signal is much stronger, appears for the longer time and has higher statistical significance between the temperature, and SSN and GAi series than between the series of temperature and volcanic sulfates (the example of such spectra can be found in the Supp. Material, Fig. S12). In the last case the areas of the coherent signal are limited by the epochs of strong volcanic eruptions (end of the 19th - beginning of the 20th c. and end of the 20th c.). Taking all this into account we conclude that the SVE effect on the atmosphere and oceans cannot be a sole reason for the (bi)-decadal oscillations found in Portuguese temperature series, especially in the middle of the 20th c., and the solar forcing still can be considered to be one of the possible sources of these particular temperature variations.

The task of separation of the multi-decadal modes associated with external solar forcing and internal atmospheric variability was fulfilled by Lohmann et al. (2004) using long instrumental data sets for SST and SLP. They showed that the spatial distributions of the multi-decadal modes of the SST and SLP variations that can be associated with solar signal are distinct from the spatial patterns of the modes related to the internal oceanic/atmospheric variability. The results of our analysis cannot be compared directly with results of Lohmann et al. (2004), however, we have to mention that there are similarities between the variations of, e.g., our spring Tmin and Tmax series (please see Supp. Material, Fig. S2-S3) and the time variations of the SST modes related to the solar signal (see solid line in Fig. 4c in Lohmann et al., 2004): the global minima/maxima of both curves take place at the same time intervals. On the other hand, our spring temperature series are ones for which the addition of the solar forcings to the MRM (see Fig. 7) significantly increase the MRM predictive power. We consider all this as an additional support for the conclusion that the Portuguese temperature series presented here have, at least for some seasons, (bi-)decadal modes of variability associated with the solar signal.

There is still a question of phase shifts between the decadal solar and temperature signals that took place around middle of the 20th c. We suppose that this shift can be related (e.g.) to the epoch of low volcanic activity (there were no large volcanic aerosol injections from about 1925 to 1963) or to the decrease of the strength of the NAO (strong westerlies and Atlantic cyclones passed at this period through the west part of Iberian Peninsula), or to the rise of the geomagnetic and solar activity. It also has to be mentioned that the NAO variations may, in turn, have imprints of the solar activity influence as was proposed in (Vaquero, 2004).

Another hypothesis is related to the excitation of the internal multidecadal modes in the atmosphere-ocean system under the influence of an external decadal signal - solar forcing. This hypothesis was proposed in, e.g., Tobias and Weiss (2000) and developed by, e.g., (Miranda et al., 2002, Raspopov et al., 2004; Gusev and Martin, 2012). It is based on the ability of a non-linear system to enhance the amplitude of its internal periodical modes under a weak forcing when "typical frequencies" of both systems are in resonance or very close to this condition (Tobias and Weiss, 2000). As was shown in Gusev and Martin (2012) such combination of the internal variability and the external forcing can results in the change of the phase between two periodicities and/or variations of the amplitude of the internal mode. This behavior is often detected in the relations between the solar forcing and climatic data and closely resembles the phase shift in our data.

\section{Conclusions}

The trends of the Tmin and Tmax measured by the Portuguese stations are different from the one observed on the more continental Spanish stations (as in e.g. del Rio et al. 2012). The Spanish data show that a faster growth of the Tmax comparing to Tmin leads to the gradual increase of the DTR, however, in the case of the Portuguese DTR series this increase changed to the decrease around the middle of the 20th c. We assume that this difference is related to the influence of the Atlantic Ocean which is stronger for the coastal Portuguese regions. 
On the whole, the response of the Tmin series to the studied forcings (anthropogenic, volcanic and solar) is stronger, clearer, seasonally more uniform and more statistically significant than one of the Tmax series. This conclusion results both from the correlation and regression analyses, and from the wavelet coherence analysis. The reason for this difference between the Tmin and Tmax lies, probably, in the strong dependence of the Tmin of the Iberian Peninsula on the radiance balance in the lower atmosphere due to dimming/brightening associated (at least partly) with circulation patterns (see del Rio et al. (2012) for the discussion). The analysis done for the shorter time period (1978-1992) by Morozova and Mironova (2015) confirm this assumption.

One of the strongest forcings for the studied period is the anthropogenic gases $\left(\mathrm{CO}_{2}\right)$. Temperature decadal variations show strong relations to the $\mathrm{CO}_{2}$ forcing. The Trend series of Tmin series correlate well with the $\mathrm{CO}_{2}$ increase. On contrary, the Tmax series shows very weak correlations with the $\mathrm{CO}_{2}$ variations. The only exception is the Tmax winter series which increase in parallel with the $\mathrm{CO}_{2}$. The $\mathrm{CO}_{2}$ effect is seen almost exclusively in the Trend components of the temperature variations.

The fingerprints of both anthropogenic and volcanic sulfates are also found in the temperature variations.

Weak but statistically significant (bi-)decadal signals in the temperature series that can be associated with the solar and geomagnetic activity variations were found. These signals are stronger during the spring and autumn seasons. The multiple regression models which include the sunspot numbers or the geomagnetic indices among other regressors have higher prediction quality. The wavelet coherence analysis shows that there are time lags between the temperature variations and the solar activity cycles. These lags are about 1-2 years in case of the 11-yr solar cycle as well as in case of the 22-yr solar magnetic cycle (relatively to the solar polar magnetic field observations). These lags are confirmed by the correlation analysis. The results obtained by these methods as well as comparison to results of other studies allow us to conclude that the found (bi-)decadal temperature variability modes can be associated, at least partly, with the effect of the solar forcing.

The results of this analysis, when confirmed by the other studies, can be applied to improve the prediction quality of the climate models that include external forcings similar to ones used here. In case the time lag between the atmospheric and solar parameters is real it can be used for forecasting purposes. On the other hand, since the mechanism of the solar influence is under discussion, the further studies are needed to understand the source(s) of such time lags.

\section{Acknowledgment}

Anna Morozova was supported by a Post-Doc FCT scholarship (Ref.: SFRH/BPD/74812/2010). CITEUC is funded by National Funds through FCT - Foundation for Science and Technology (project: UID/Multi/ 00611/2013) and FEDER - European Regional Development Fund through COMPETE 2020 - Operational Programme Competitiveness and Internationalization (project: POCI-01-0145-FEDER-006922). The authoresses are grateful to Anonymous Reviewers for their useful comments and recommendations that helped to improve the quality of the manuscript.

\section{Appendix A. Seasonal-Trend decomposition procedure based on LOESS.}

The STL decomposition was introduced and developed by (Cleveland, 1979, Cleveland and Devlin, 1988; Cleveland et al.,
1990). It consists of two recursive sequences of calculations: outer and inner loops each of which can be passed a number of times. The inner loop divides the original series $X(t)$ into trend $T(t)$ and cyclic $C_{n p}(t)$ components (with a predefined period $n p$ ) in a sequence of detrending, smoothing (LOESS and moving average) and filtering procedures. On the whole, the STL can be considered as a consecutive application of low(high)-pass and band-pass filters. A number of parameters allows one to choose the level of smoothing and to limit the periodicities that are too long-term to be included in the cyclic component. The outer loop is used to calculate (1) robustness weights which are used in the LOESS smoothing of the next sequence of inner loop runs and (2) a convergence criterion which defines when iteration process has to stop. The STL inner loop consists of 6 main steps:

Step 1. Detrending of the original series using the trend component $T^{k-1}(t)$ calculated in the previous step (for the first run $T^{0}(t)$ $=0)$ :

$L_{1}^{k}(t)=X(t)-T^{k-1}(t)$

Step 2. The detrended series $L_{1}{ }^{k}(t)$ is divided into a number of cycle-subseries (each has a length $n p$ ). Afterwards, these subseries are smoothed using the LOESS smoothing procedure which is explained in details in the Appendix B. After the smoothing, all cycle-subseries are combined again into one temporary cyclic series $C_{n p 1^{k}}{ }^{k}(t)$. This series is longer than the original by $n p$.

Step 3. Low-pass filtering is applied to smooth and shorten $C_{n p}{ }^{k}$ $(t)$ series. It consists of two consequent moving averaging of length $n p$, followed by moving averaging of length 3 , followed by the LOESS smoothing. At the end of this step the new temporary cyclic series $L^{k}(t)$ have same length as the original series.

Step 4. The $C_{n p}{ }^{k}(t)$ series is detrended to remove low-frequency variations from the cyclic component:

$C_{n p}{ }^{k}(t)=C_{n p 1}{ }^{k}(t)-L^{k}(t)$

This detrended series $C_{n p}{ }^{k}(t)$ is a final cyclic components of the current run of the internal loop.

Step 5. Deseasonalized series is constructed as

$T_{1}^{k}(t)=X(t)-C_{n p}{ }^{k}(t)$

Step 6. Deseasonalized series $T_{1}{ }^{k}(t)$ is smoothed by LOESS giving the final trend component $T^{k}(t)$ of the current run of the internal loop.

The outer loop starts with calculating of residuals $R^{k}(t)$ (Eq. (A4)) and robustness weights that diminish the influence of outliers in $R^{k}(t)$ for the next runs of the inner loop (see Cleveland et al., 1990):

$R^{k}(t)=X(t)-C_{n p}{ }^{k}(t)-T^{k}(t)$

The outer loop can be run a predefined number of times (large enough to ensure robustness of the decomposition procedure), or a convergence criterion could be used to stop the outer loop procedure when a certain level of convergence in achieved. In this study we used the convergence criterion proposed by (Cleveland et al., 1990): 
$\max _{t} \mid U^{k}(t)-U^{k-1}(t) I /\left(\max _{t} U^{k}(t)-\min _{t} U^{k}(t)\right)<0.01$

where $U^{k}(t)$ is a result of kth iteration of either trend or seasonal component. We applied this criterion first to the trend component and afterwards, in case of convergence, to cyclic component. The decomposition stops when both trend and cyclic components satisfy the convergence criterion.

The STL is simple for the computer implementation; extracted components are robust and easy to interpret. This method can be used both as a low(high)-pass and as a band-pass filter depending on the purpose of the study and the characteristic period $n p$. In our case, the analysis of the Trend components calculated using different $n p$ values shows that they are very similar to results of smoothing of the original series by the moving averaging with a certain window length: about 5 years for $n p=1$, about 25 years for $n p=10-11$ and about 50 years for $n p=20-22$. However, the use of the STL has at least one advantage comparing to the moving average smoothing: it allows one to get a smoothed series of the same length as the original one.

\section{Appendix B. LOESS smoothing procedure}

In the LOESS (LOcally wEighted regreSSion) procedure the smoothed series is calculated using a regression function defined along all possible scale of independent value. It is thoroughly described in (Cleveland, 1979, Cleveland and Devlin, 1988; Cleveland et al., 1990). Here we present a short description.

Let $Y\left(t_{i}\right)$ be a time dependent variable defined for $t_{i}=t_{1} \ldots t_{n}$. A positive integer, $q$, is chosen. For any given $t$, a neighborhood weight $v_{i}$ is calculated (Eq. B1) using the tricube weight function $W$ (Eq. $\mathrm{B} 2)$.

$v_{i}=W\left(\left|t_{i}-t\right| / \lambda_{q}(t)\right)$

$W(u)=\left[\begin{array}{l}\left(1-u^{3}\right), 0 \leq u<1 ; \\ 0, u \geq 1\end{array}\right.$

Here $\lambda_{q}(t)$ is the distance of the $q$ th farthest $t_{i}$ from $t$ :

$\lambda_{q}(t)=\left[\begin{array}{l}\left|t_{q}-t\right|, q \leq n ; \\ \left|t_{n}-t\right| \frac{q}{n}, q>n ;\end{array}\right.$

If the robustness weights mentioned in the Appendix A are non-zero, the neighborhood weights are multiplied by the robustness weights. The neighborhood weights are used in the locally-fitting procedure for $Y\left(t_{i}\right)$. The fitting procedure use polynomial function of degree $d$. Cleveland et al. (1990) propose to use locally-linear $(d=0$ or $d=1)$ or locally-quadratic $(d=2)$ fitting. The coefficients of the polynomial function are calculated on the base of the $Y\left(t_{i}\right)$ data using the least-square technique. The value of locally-fitting polynomial at $t$ is the value of the LOESS regression at this point.

The main advantage of the LOESS smoothing procedure is that it can be defined everywhere and not just at $t_{i}$. Thus, the LOESS smoothing can (1) be applied to the series with missing values, (2) successfully smooth a series without loss of parts near to the beginning and the end of the series. The STL effectively uses the second property of the LOESS on its Steps 2 and 3 (see Appendix A).

\section{Appendix C. Supplementary material}

Supplementary data associated with this article can be found in the online version at http://dx.doi.org/10.1016/j.jastp.2016.02.006.

\section{References}

Barlyaeva, T.V., 2013. External forcing on air-surface temperature: geographical distribution of sensitive climate zones. J. Atmos. Sol. -Terr. Phys. 94, 81-92.

Barlyaeva, T.V., Mironova, I.A., Ponyavin, D.I., 2009. Nature of decadal variations in the climatic data of the second half of the 20th century. Doklady Earth Sci. 425A (3), 419-423.

Bjornsson, H., Venegas, S.A., 1997. A manual for EOF and SVD analyses of climatic data. McGill University (97-1).

Clette, F., Svalgaard, L., Vaquero, J.M., Cliver, E.W., 2014. Revisiting the Sunspot Number. Space Sci. Rev. 186 (1), 35-103. http://dx.doi.org/10.1007/ s11214-014-0074-2.

Clette, F., Cliver, E.W., Lefèvre, L., Svalgaard, L., Vaquero, J.M., 2015. Revision of the Sunspot Number(s). Space Weather 13 (9), 529-530. http://dx.doi.org/10.1002/ 2015 SW001264.

Cleveland, W.S., 1979. Robust locally weighted regression and smoothing scatterplots. J. Am. Stat. Assoc. 74 (368), 829-836.

Cleveland, W.S., Devlin, S.J., 1988. Locally weighted regression: An approach to regression analysis by local fitting. J. Am. Stat. Assoc. 83 (403), 596-610.

Cleveland, R.B., Cleveland, W.S., McRae, J.E., Terpenning, I., 1990. STL: a seasonaltrend decomposition procedure based on LOESS. J. Off. Stat. 6, 3-73.

Cliver, E.W., Boriakoff, V., Bounar, K.H., 1996. The 22-year cycle of geomagnetic and solar wind activity. J. Geophys. Res. 101 (A12), 27091-27109. http://dx.doi.org/10.1029/ 96JA02037.

del Río, S., Cano-Ortiz, A., Herrero, L., Penas, A., 2012. Recent trends in mean maximum and minimum air temperatures over Spain (1961-2006). Theor. Appl. Climatol. 109, 605-626. http://dx.doi.org/10.1007/ s00704-012-0593-2.

Durre, I., Wallace, J.M., 2001. Factors influencing the cold-season diurnal temperature range in the United States. J. Clim. 14 (15), 3263-3278.

Ebisuzaki, W., 1997. A method to estimate the statistical significance of a correlation when the data are serially correlated. J. Clim. 10 (9), 2147-2153.

Erlykin, A.D., Sloan, T., Wolfendale, A.W., 2010. Correlations of clouds, cosmic rays and solar irradiation over the Earth. J. Atmos. Sol. -Ter. Phys. 72 (2-3), 151-156.

Fröhlich, C., 2012. Total solar irradiance observations. Surv. Geophys. 33 (3-4), $453-473$.

Gámiz-Fortis, S.R., Esteban-Parra, M.J., Pozo-Vázquez, D., Castro-Díez, Y., 2011. Variability of the monthly European temperature and its association with the Atlantic sea-surface temperature from interannual to multidecadal scales. Int. J. Climatol. 31 (14), 2115-2140.

Gómez-Navarro, J.J., Montávez, J.P., Jimenez-Guerrero, P., Jerez, S., García-Valero, J. A., González-Rouco, J.F., 2010. Warming patterns in regional climate change projections over the Iberian Peninsula. Meteorol. Z. 19 (11), 275-285. http://dx. doi.org/10.1127/0941 - 2948/2010/0351.

Gómez-Navarro, J.J., Montávez, J.P., Jimenez-Guerrero, P., Jerez, S., Lorente-Plazas, R., Gonzalez-Rouco, J.F., Zorita, E., 2012. Internal and external variability in regional simulations of the Iberian Peninsula climate over the last millennium. Clim. Past 8, 25-36.

Gómez-Navarro, J.J., Montávez, J.P., Wagner, S., Zorita, E., 2013. A regional climate palaeosimulation for Europe in the period 1500-1990 - Part 1: model validation. Clim. Past 9, 1667-1682. http://dx.doi.org/10.5194/cp-9-1667-2013.

Gray, L.J., Scaife, A.A., Mitchell, D.M., Osprey, S., Ineson, S., Hardiman, S., Butchart, N., Knight, J., Sutton, R., Kodera, K., 2013. A lagged response to the 11 year solar cycle in observed winter Atlantic/European weather patterns. J. Geophys. Res. Atmos. 118 (13), 405-413. http://dx.doi.org/10.1002/2013JD020062.

Gusev, A.A., Martin, I.M., 2012. Possible evidence of the resonant influence of solar forcing on the climate system. J. Atmos. Sol. -Terr. Phys. 80, 173-178.

Hannachi, A., Jolliffe, I.T., Stephenson, D.B., 2007. Empirical orthogonal functions and related techniques in atmospheric science: a review. Int. J. Climatol. 27 (9), 1119-1152.

Hegerl, G.C., Crowley, T.J., Allen, M., Hyde, W.T., Pollack, H.N., Smerdon, J., Zorita, E. 2007. Detection of human influence on a new, validated 1500 -year temperature reconstruction. J. Clim. 20, 650-666. http://dx.doi.org/10.1175/JCLI4011.1.

Hegerl, G., Luterbacher, J., González-Rouco, F., Tett, S.F.B., Crowley, T., Xoplaki, E., 2011. Influence of human and natural forcing on European seasonal temperatures. Nat. Geosci. 4, 99-103.

Heino, R., Brazdil, R., Forland, E., Tuomenvirta, H., Alexandersson, H., Beniston, M. Pfister, C., Rebetez, M., Rosenhagen, G., Rosner, S., 1999. Progress in the study of climatic extremes in Northern and Central Europe. Clim. Change 42 (1), $151-181$.

Hurrell, J., National Center for Atmospheric Research Staff (Eds). The Climate Data Guide: Hurrell North Atlantic Oscillation (NAO) Index (PC-based). Last modified 15 Oct 2012. On-line: 〈http://climatedataguide.ucar.edu/guidance/hurrellnorth-atlantic-oscillation-nao-index-pc-based $\rangle$.

Karl, T.R., Jones, P.D., Knight, R.W., Kukla, Ge, Plummer, N., Razuvayev, V., Gallo, K.P., Lindseay, J., Charlson, R.J., Peterson, T.C., 1993. A new perspective on recent global warming: asymmetric trends of daily maximum and minimum temperature. Bull. Am. Meteorol. Soc. 74; , pp. 1007-1024.

Künsch, H.R., 1989. The jackknife and the bootstrap for general stationary observations. Ann. Stat. 17, 1217-1241.

Lahiri, S.N., 1999. Theoretical comparisons of block bootstrap methods. Ann. Stat. 27, 386-404.

Lam, M.M., Tinsley, B.A., 2015. Solar wind-atmospheric electricity-cloud microphysics connections to weather and climate. J. Atmos. Sol. -Ter. Phys. http://dx. doi.org/10.1016/j.jastp.2015.10.019 
Lean,J., Rind, D.H., 1889 to 2006. How Natural and Anthropogenic Influences Alter Global and Regional Surface Temperatures: GRL 35, 18, CiteID L18701.

Liu, S.C., Wang, C.-H., Shiu, C.-J., Chang, H.-W., Hsiao, C.-K., Liaw, S.-H., 2002. Reduction in sunshine duration over Taiwan: causes and implications. Trop. Atmos. Ocean Proj. 13 (4), 523-545.

Lockwood, M., 2001. Long-term variations in the magnetic fields of the Sun and the heliosphere: their origin, effects, and implications. J. Geophys, Res. 106 (A8), $16021-16038$.

Lockwood, M., 2012. Solar influence on global and regional climates. Surv. Geophys. $33(3-4), 503-534$.

Lockwood, M., Fröhlich, C., 2007. Recent oppositely directed trends in solar climate forcings and the global mean surface air temperature. Proc. R. Soc. A-Math. Phys. 463 (2086), 2447-2460.

Lohmann, G., Rimbu, N., Dima, M., 2004. Climate signature of solar irradiance variations: analysis of long-term instrumental, historical, and proxy data. Int. J. Climatol. 24 (8), 1045-1056.

Lukianova, R., Alekseev, G., 2004. Long-term correlation between the NAO and solar activity. Sol. Phys. 224 (1-2), 445-454.

Luterbacher, J., Dietrich, D., Xoplaki, E., Grosjean, M., Wanner, H., 2004. European seasonal and annual temperature variability, trends, and extremes since 1500 . Science 303, 1499-1503. http://dx.doi.org/10.1126/science.1093877.

Makowski, K., Wild, M., Ohmura, A., 2008. Diurnal temperature range over Europe between 1950 and 2005. Atmos. Chem. Phys. 8 (21), 6483-6498.

Mann, M.E., Gille, E.P., Bradley, R.S., Hughes, M.K., Overpeck, J., Keimig, F.T., Gross, W., 2000a. Global Temperature Patterns in Past Centuries: An Interactive Presentation. IGBP Pages/World Data Center for Paleoclimatology, Data Contribution Series \#2000 - 075., NOAA/NGDC Paleoclimatology Program, Boulder CO, USA. On-line: 〈http://www.ncdc.noaa.gov/paleo/ei/〉.

Mann, M.E., Gille, E.P., Bradley, R.S., Hughes, M.K., Overpeck, J., Keimig, F.T., Gross, W., 2000b. Global temperature patterns in past centuries: an interactive presentation. Earth Interact. 4, 4.

Maraun, D., Kurths, J., 2004. Cross wavelet analysis: significance testing and pitfalls Nonlin. Process. Geophys. 11, 505-514.

McConnell, J.R., Edwards, R., Kok, G.L., Flanner, M.G., Zender, C.S., Saltzman, E.S. Banta, J.R., Pasteris, D.R., Carter, M.M., Kahl, J.D.W., 2007a. Greenland D4 Ice Core Black Carbon, VA, and nssS Data. IGBP PAGES/World Data Center for Paleoclimatology Data Contribution Series \# 2007 - 060, NOAA/NCDC Paleoclimatology Program, Boulder CO, USA. On-line: 〈http://www.ncdc.noaa.gov/pa leo/icecore/greenland/greenland.html〉.

McConnell, J.R., Edwards, R., Kok, G.L., Flanner, M.G., Zender, C.S., Saltzman, E.S., Banta, J.R., Pasteris, D.R., Carter, M.M., Kahl, J.D.W., 2007b. 20th-century industrial black carbon emissions altered arctic climate forcing. Science 317 1381-1384.

Meehl, G.A., Washington, W.M., Wigley, T.M.L., Arblaster, J.A., Dai, A., 2003. Solar and greenhouse gas forcing and climate response in the twentieth century. J. Clim. 16, 426-444.

Meyers, S.R., Pagani, M., 2006. Quasi-periodic climate teleconnections between northern and southern Europe during the 17th-20th centuries. Global Planet. Change 54 (3), 291-301.

Miranda, P., Coelho, F.E.S., Tomé, A.R., Valente, M.A., Carvalho, A., Pires, C., Pires, H. Pires, O.V.C., Ramalho, C., 2002. 20th century Portuguese climate and climate scenarios, In: Santos, F.D., Forbes, K., Moita, R. (Eds.) Climate Change in Portugal: Scenarios, Impacts and Adaptation Measures (SIAM Project), Gradiva, pp. 23-83.

Mironova, I.A., Aplin, K.L., Arnold, F., Bazilevskaya, G.A., Harrison, R.G., Krivolutsky, A.A., Nicoll, K.A., Rozanov, E.V., Turunen, E., Usoskin, I.G., 2015. Energetic particle influence on the Earth's Atmosphere. Space Sci. Rev. 194, 1-96. http://dx doi.org/10.1007/s11214-015-0185-4.

Morozova, A.L., Mironova, I.A., 2015. Aerosols over continental Portugal (19781993): their sources and an impact on the regional climate. Atmos. Chem. Phys. 15 (11), 6407-6418.

Morozova, A.L., Valente, M.A., 2012. Homogenization of Portuguese long-term temperature data series: Lisbon, Coimbra and Porto. Earth Syst. Sci. Data 4 $187-213$

Mursula, K., Usoskin, I.G., Kovaltsov, G.A., 2001. Persistent 22-year cycle in sunspot activity: Evidence for a relic solar magnetic field. Solar Phys. 198 (1), 51-56.

PAGES $2 \mathrm{k}$ Consortium, 2013k. Continental-scale temperature variability during the past two millennia. Nat. Geosci. 6, 339-346.

Palamara, D., Bryant, E., 2004. Geomagnetic activity forcing of the Northern Annular Mode via the stratosphere. Ann. Geophys. 22 (3), 725-731.

Raspopov, O.M., Dergachev, V.A., Kolström, T., 2004. Hale cyclicity of solar activity and its relation to climate variability. Sol. Phys. 224 (1-2), 455-463.

Reichler, T., Kim, J., Manzini, E., Kröger, J., 2012. A stratospheric connection to Atlantic climate variability. Nat. Geosci. 5 (11), 783-787.

Robertson, A., Overpeck, J., Rind, D., Mosley-Thompson, E., Zielinski, G., Lean, J., Koch, D., Penner, J., Tegen, I., Healy, R., 2001a. Hypothesized Climate Forcing Time Series for the Last 500 Years, IGBP PAGES/World Data Center for Paleoclimatology Data Contribution Series \#2001 - 057, NOAA/NGDC Paleoclimatology Program, Boulder CO, USA. On-line: 〈http://www.ncdc.noaa.gov/paleo/ pubs/robertson2001/robertson2001.html〉.

Robertson, A., Overpeck, J., Rind, D., Mosley-Thompson, E., Zielinski, G., Lean, J. Koch, D., Penner, J., Tegen, I., Healy, R., 2001b. Hypothesized climate forcing time series for the last 500 years. J. Geophys. Res. Atmos. 106 (D14), $14,783-14,803$.

Rozas, V., García-González, I., 2012. Non-stationary influence of El Niño-Southern Oscillation and winter temperature on oak latewood growth in NW Iberian Peninsula. Int. J. Biometeorol. 56, 787-800.

Sánchez-Lorenzo, A., Brunetti, M., Calbó, J., Martín-Vide, J., 2007. Recent spatial and temporal variability and trends of sunshine duration over the Iberian Peninsula from a homogenized data set. J. Geophys. Res. -Atmos. 112, D20115.

Sánchez-Lorenzo, A., Pereira, P., Lopez-Bustins, J.A., Lolis, C.J., 2012. Summer nighttime temperature trends on the Iberian Peninsula and their connection with large-scale atmospheric circulation patterns. Int. J. Climatol. 32 (9), 1326-1335.

Sato, M., Hansen, J.E., McCormick, M.P., Pollack, J.B., 1993. Stratospheric aerosol optical depth, 1850 - 1990. J. Geophys. Res. 98, 22987-22994.

Shlens, J., 2009. A Tutorial on Principal Component Analysis. Systems Neurobiology Laboratory. University of California at San Diego, version 3.01. On-line 〈http:// snl.salk.edu/ shlens/pca.pdf .

Smith,S.J., van Aardenne, J., Klimont, Z., Andres, R.J., 2011. Anthropogenic Sulfur Dioxide Emissions, 1850-2005: National and Regional Data Set by Source Category, Version 2.86. Palisades, NY: NASA. On-line: http://sedac.ciesin.columbia. edu/data/set/haso2-anthro-sulfur-dioxide-emissions - 1850 - 2005-v2 - 86 .

Svalgaard, L., Cliver, E.W., 2005. The IDV index: its derivation and use in inferring long-term variations of the interplanetary magnetic field strength. J. Geophys. Res. 110 (A12103), 1-9.

Svalgaard, L., Cliver, E.W., Le Sager, P., 2004. IHV: a new long-term geomagnetic index. Adv. Space Res. 34 (2), 436-439.

Tinsley, B.A., 2008. The Global Atmospheric Electric Circuit and its Effect on Cloud Microphysics, Report on Progress in Physics, 71, 066801.

Tobias, S.M., Weiss, N.O., 2000. Resonant interactions between solar activity and climate. J. Clim. 13 (21), 3745-3759.

Torrence, C., Compo, G.P., 1998. A practical guide to wavelet analysis. Bull. Am. Meteor. Soc. 79, 61-78.

Usoskin, I.G., Mironova, I.A., Korte, M., Kovaltsov, G.A., 2010. Regional millennial trend in the cosmic ray induced ionization of the troposphere. J. Atmos. SolarTerr. Phys. 72 (1), 19-25.

Vaquero, J.M., 2004. Solar signal in the number of floods recorded for the Tagus river basin over the last millennium. Clim. Change 66 (1), 23-26.

Voiculescu, M., Usoskin, I.G., Mursula, K., 2006. Different response of clouds to solar input. Geophys. Res. Lett. 33 (L21802), 1-5.

Weber, R.O., Talkner, P., Stefanicki, G., 1994. Asymmetric diurnal temperature change in the Alpine region. Geophys. Res. Lett. 21 (8), 673-676.

Zanchettin, D., Timmreck, C., Bothe, O., Lorenz, S.J., Hegerl, G., Graf, H.-F., Luterbacher, J., Jungclaus, J.H., 2013. Delayed winter warming: a robust decadal response to strong tropical volcanic eruptions? Geophys. Res. Lett. 40 (1) 204-209. http://dx.doi.org/10.1002/GRL.20060.

Zanchettin, D., Timmreck, C., Graf, H.-F., Rubino, A., Lorenz, S., Lohmann, K., Krüger, K., Jungclaus, J.H., 2012. Bi-decadal variability excited in the coupled oceanatmosphere system by strong tropical volcanic eruptions. Clim. Dyn. 39 (1-2), 419-444. 